\title{
Synopsis of Acalypha L. (Euphorbiaceae) of Peru and Bolivia, with description of a new species
}

\author{
José María Cardiel ${ }^{1 *}$, Michael Nee² \& Pablo Muñoz Rodríguez ${ }^{1}$ \\ ${ }^{1}$ Departamento de Biología, Facultad de Ciencias, Universidad Autónoma de Madrid, Ciudad Universitaria de Cantoblanco, \\ Code 28049, Madrid, Spain; jm.cardiel@uam.es \\ ${ }^{2}$ The New York Botanical Garden, 2900 Southern Boulevard, Bronx, New York, 10458, USA
}

\begin{abstract}
Cardiel, J.M., Nee, M. \& Muñoz Rodríguez, P. 2013. Synopsis of Acalypha L. (Euphorbiaceae) of Peru and Bolivia, with description of a new species. Anales Jard. Bot. Madrid 70(2): 152-177.

A critical review of the Peruvian and Bolivian species of Acalypha L. (Euphorbiaceae) is presented. As a result 41 species (42 taxa) are accepted, 31 from Peru and 28 from Bolivia, and 94 names are considered synonyms. A new species is described and illustrated, $A$. psamofila, based on Bolivian material. Comprehensive nomenclatural information is supplied and 36 lectotypes and one neotype are designated. An identification key is also provided, as well as original illustrations of six species and distribution maps of all the accepted species.
\end{abstract}

Keywords: floristic, lectotypification, Malpighiales, Neotropics, taxonomy.

\section{INTRODUCTION}

Acalypha L is a monophyletic genus belonging to subfamily Acalyphoideae Kunth ex Beilschm., the most diverse and complex in the family Euphorbiaceae Juss. (Hayden \& Hayden, 2000; Tokuoka, 2007; Wurdack \& Davis, 2009). With c. 500 species Acalypha is, after Euphorbia L. and Croton L., the third largest genus in the Euphorbiaceae. The genus is found in tropical and subtropical regions worldwide in a variety of habitats from tropical rainforests to subdesertic areas, and from sea level to 4000 meters of elevation. The Neotropics are home to around two-thirds of the species.

Acalypha has traditionally been a poorly known genus compared with Euphorbia and Croton. The last global treatment was made by Pax and Hoffmann (1924) in Engler's Pflanzenreich. The knowledge of Acalypha in the Americas has been increasing during the last 20 years through updated national floristic treatments and checklists, e.g., Argentina (Bacigalupo \& Mulgura, 1999), Belize (Balick \& al., 2000), Brazil (Cardiel, 2010), Colombia (Cardiel, 1995; Murillo, 2004), Costa Rica (Burger \& Huft, 1995; González, 2010), Ecuador (Cardiel \& Muñoz, 2012a), Guianas (Gillespie, 1993, 1997), Honduras (Nelson, 2008), Mexico (Steinmann, 2002), Nicaragua (Levin, 2001), Panama (Correa \& al., 2004), South Cone (Berry, 2007), and Venezuela (Ambruster \& al., 2007; Cardiel, 1999; Levin, 1999, 2008).

Peruvian Acalypha have been treated nationally in Macbride's (1951) and in Brako's (1993) checklist, updated by Ulloa Ulloa \& al. (2004). Several regional or thematic floras also included Acalypha species: León \& al. (2006), Pennington \& al. (2004), Valenzuela \& al. (2007) and Vásquez $(1997,2010)$. Taxonomic works focused on Peruvian Aca-

\section{Resumen}

Cardiel, J.M., Nee, M. \& Muñoz Rodríguez, P. 2013. Sinopsis de Acalypha L. (Euphorbiaceae) de Perú y Bolivia, con la descripción de una nueva especie. Anales Jard. Bot. Madrid 70(2): 152-177 (en inglés).

Se presenta una revisión crítica de las especies de Acalypha L. (Euphorbiaceae) presentes en Bolivia y Perú. Como resultado se reconocen 41 especies (42 táxones), 31 para Perú y 28 para Bolivia, y 94 nombres son considerados sinónimos. Se describe e ilustra una nueva especie, A. psamofila, basada en material boliviano. Se aporta una exhaustiva información nomenclatural y se designan 36 lectótipos y un neótipo. Se incluye también una clave de identificación, así como ilustraciones originales de seis especies y mapas de distribución de todas las especies aceptadas.

Palabras clave: florística, lectotipificación, Malpighiales, Neotrópico, taxonomía.

lypha include those of Cardiel (2003, 2006, 2007); with 32 species included in Cardiel (2007). The Bolivian flora is less well known. There is no national flora, only Foster's (1958) list of names, which includes 55 Acalypha names and 41 accepted species, and the unpublished checklist of Jørgensen \& al. (in preparation). Regional or thematic floras that mentioned Acalypha species include Arroyo \& Villarroel (2009), Jardim \& al. (2003), Killeen \& al. (1993), Killeen \& Schulenberg (1998), Moraes (1990), Parker \& Bailey (1991), Serrano \& Terán (1998[2000]) and Vargas (1993). Several new Bolivian species of Acalypha have been described recently (Cardiel, 2006; Cardiel \& Muñoz, 2012b). Also relevant is the recent nomenclatural reorganization of Acalypha sect. Communes (Cardiel \& al., 2013). This section includes three Bolivian species: A. communis, $A$. variabilis and $A$. psamofila, described here.

We present here a critical synopsis of the Peruvian and Bolivian species of Acalypha, with a key to their identification. Six original illustrations are included, as well as synoptic distribution maps of all the species.

\section{MATERIAL AND METHODS}

This work is based on the study of 1175 collections of Acalypha from Peru and 830 collections from Bolivia, from the following herbaria: A, AAU, B, BM, BP, BR, C, CAS, COL, DAV, F, G, GB, GH, GOET, HAL, HBG, JE, K, L, LE, LPB, M, MA, MO, MPU, NY, P, PR, S, SEL, SI, U, UC, UPS, US, W, WU, WRSL, Z and ZT (acronyms according to Thiers, 2013). We found a large number of type specimens, clarifying the identity of many names. Typifications were made after a carefully review of the original literature on the 
taxa and examination of the nomenclatural types. Where no holotype was indicated, or it has been lost or destroyed, a lectotype or neotype is designated according to the International Code of Nomenclature for Algae, Fungi and Plants (ICN, McNeill \& al., 2012).

The structure of the catalogue follows, in general terms, the one we employed in previous works (Cardiel \& Muñoz, 2012a; Muñoz-Rodríguez \& al., 2014). The accepted species are cited in alphabetical order, including original publications, and synonyms based on Peruvian and Bolivian specimens. We cite the type collection for each name, indicating the specimens we studied with an exclamation mark (!). We cite an illustration when available. Then we summarize the general geographic distribution following TDWG standards (Brummitt, 2001), and the detailed Peruvian and Bolivian distribution, indicating the departments were the species have been found in each country; this information is also reflected in the distribution maps provided. We also indicate the total number of collections reviewed for each taxon; detailed information about these collections can be consulted in the data resources cited. Biogeographic regions [following, in general terms, the natural regions proposed by Brako \& Zarucchi (1993) for Peru and those proposed by Renvoize (1998) for Bolivia], elevation range, and habitat are then described; this information was obtained exclusively from the studied specimens. We then list recent literature and thereafter we include, when needed, any other relevant information, including justifications for nomenclatural decisions. For the new species described we provide a more detailed information, including a diagnosis and full description, as well as a discussion and comments on the systematics of related species.

\section{Data resources}

All the information gathered as part of this work is available online (Cardiel \& al., 2013). In addition, the information of studied specimens has been also uploaded to the Global Biodiversity Information Facility (GBIF) (http://data.gbif. org/datasets/resource/12046/).

\section{RESULTS}

We record 137 scientific names related to Acalypha from Peru and Bolivia, 41 of which are accepted species, 31 from Peru and 28 from Bolivia (Appendix 1). 94 names are considered synonyms, and four species are considered doubtful or excluded. We designate 36 lectotypes and one neotype. A new species, $A$. psamofila, is described, based on Bolivian material. Five species are endemic to Peru $(A$. argomuelleri, $A$. aronioides, $A$. delicata, $A$. salicina and $A$. simplicistila), and four are endemic to Bolivia (A. beckii, A. machiensis, $A$. neeana and $A$. psamofila). Three species are known only from these two countries: A. bibiscifolia, A. reflexa and A. peruviana. 18 species are present in both countries, and two species are introduced: $A$. hispida and $A$. wilkesiana. Finally, four taxa are treated as doubtful and excluded names: $A$. brachyclada, A. contermina, A. cuprea and A. jubifera.

\section{KEY TO ACALYPHA OF PERU AND BOLIVIA}

\section{Key to the subgenera}

1. Female flowers pedicellate, calyx with 4 or 5 sepals, the subtending bracts inconspicuous, not becoming foliaceous in fruit

Subgen. Linostachys

2. Female flowers sessile, calyx with 3 sepals, the subtending bracts becoming foliaceous and accrescent in fruit (except in A. hispida)

Subgen. Acalypha

\section{Key to the species}

\section{Subgenus Linostachys}

1a. Leaf blade palmately nerved, brightly colored minute resinous droplets present, mainly on lower leaf surface ......... 40. A. villosa

1b. Leaf blade pinnately nerved, resinous droplets absent ................. 2

2a. Female inflorescences paniculate; leaf blades with 10-17 veins per side; petioles generally more than $1 \mathrm{~cm}$ long .... 25. A. platyphylla

$2 b$. Female inflorescences racemose; leaf blades with 9-13 veins per side; petioles less than $1 \mathrm{~cm}$ long

30. A. salicifolia

\section{Subgenus Acalypha}

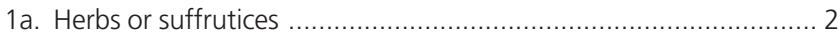

1b. Trees or shrubs .................................................................... 15

2a. Female inflorescences densely flowered, ellipsoid or cylindrical, with the axis completely covered by the bracts, not conspicuously visible

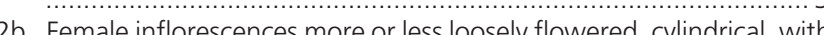
the axis conspicuously visible between the bracts ....................... 7

3a. Female bracts with long awned lobes ..................................... 4

3b. Female bracts with triangular awnless lobes ............................ 5

4a. Young branches and leaves with glandular hairs; leaf blades acuminate at apex; female inflorescences axillary and terminal; styles unbranched ................................................. 5. A. alopecuroidea

4b. Young branches and leaves without glandular hairs; leaf blades acute at apex; female inflorescences axillary; styles branched ...........

2. A. arvensis

5a. Inflorescences ellipsoid, unisexual or androgynous (mostly female with a distal segment of male flowers) .................... 27. A. poiretii

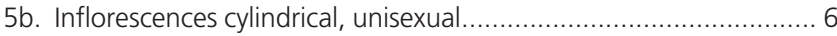

6 a. Female bracts with 3-6 teeth ca. of the bract length

17. A. infesta

6b. Female bracts with $9-13$ teeth less than $1 / 4$ of the bract length.

7. A. boliviensis

7a. Annual herbs or suffrutices; inflorescences unisexual; female inflorescences terminal; sometimes solitary, ebracteate female flowers present

7b. Usually suffrutices; some inflorescences unisexual, some androgynous (mostly male with one to several female flowers at base), terminal or axillary; solitary, ebracteate female flowers absent ........ 14

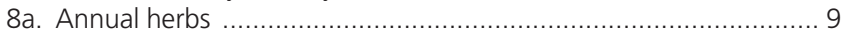

8b. Suffrutices ................................................................. 10

9a. Ovaries hispid; stipules up to $5 \mathrm{~mm}$ long, with rigid hairs to $1.5 \mathrm{~mm}$.

33. A. schultesii

9b. Ovaries glabrous; stipules minute, 1-1.5 mm long, ciliated or hipidulous, without rigid hairs

38. A. subcastrata

10a. Male inflorescences terminal; female inflorescences terminal; some female flowers solitary, axillary, without developed bracts......

14. A. herzogiana

10b. Male inflorescences axillary; female inflorescences terminal or axillary; solitary female flowers absent ...................................... 11

11a. Leaf blades usually conduplicate, glabrous or subglabrous, lower leaf surface covered by a bright exudate, sometime reduced to spots, bright resinous droplets present or absent; female bracts without glandular hairs ................................................. 39. A. variabilis

11b. Leaf blades not conduplicate, more or less densely pubescent to velutinous, lower leaf surface not covered by bright exudate, usually with conspicuous bright resinous droplets; female bracts with or without glandular hairs

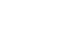
.

.


12a. Young branches and lower leaf surface with whitish indument; leaf blade obtuse; female bracts without glandular hairs

28. A. psamofila

12b. Young branches and lower leaf surface without whitish indument; leaf blade acute to acuminate; female bracts with glandular hairs....

13a. Leaf blades firm-membranaceous, velutinous; petioles $3-5 \mathrm{~cm}$ long ... 8a. A. communis subsp. communis

13b. Leaf blades thin-membranaceous, puberulous, promptly glabrescent; petioles 3-14(-18) cm long

8b. A. communis subsp. saltensis

14a. Male inflorescences terminal; developed female bracts without glandular hairs

21. A. multicaulis

14b. Male inflorescences axillary; developed female bracts with glandular hairs

10. A. cuspidata

15a. Inflorescences unisexual or rarely some terminal inflorescences androgynous, mostly female with a distal segment of male flowers

15b. Inflorescences androgynous, mostly male with one to several female flowers at the base, others male ......................................... 32

16a. All female inflorescences axillary ............................................. 17

16b. Some female inflorescences terminal ....................................... 23

17a. Leaf blade pinnately nerved (rarely pinnipalmately in A. scandens) ...

17b. Leaf blade palmately nerved .............................................. 20

18a. Shrubs, usually clambering; female inflorescences $25-40 \mathrm{~cm}$ long ...

32. A. scandens

18b. Trees or shrubs, not clambering; female inflorescences $7-15 \mathrm{~cm}$ long

19a. Leaf blades finely membranous, ovate-lanceolate or elliptic-lanceolate the base rounded to acute; female bracts with glandular hairs

11. A. delicata

19b. Leaf blades sub-chartaceous, generally obovate, the base subcuneate; female bracts without glandular hairs ........ 9. A. cuneata

20a. Leaves with stellate hairs ............................... 12. A. dictyoneura

20b. Leaves without stellate hairs ................................................. 21

21a. Female inflorescences extremely densely flowered, with the axis hidden; bracts non-accrescent; male inflorescences absent (usually cultivated plants)

16. A. hispida

$21 \mathrm{~b}$. Female inflorescences more or less densely flowered, with the axis visible; bracts conspicuously accrescent; male inflorescences usually present

22

22a. Leaf blades generally variegated; female inflorescences up to $10 \mathrm{~cm}$ long (usually cultivated plants)

41. A. wilkesiana

22b. Leaf blades not variegate; female inflorescences more than $15 \mathrm{~cm}$ long ......................................................... 20. A. macrostachya

23a. Trees or shrubs; female bracts more or less deeply divided into triangular or lanceolate teeth, acute to cuspidate at apex ................ 24

23b. Shrubs (or suffrutices); female bracts deeply divided into linear teeth, usually rounded at apex

24a. Female bracts with glandular hairs ........................................ 25

24b. Female bracts without glandular hairs ........................................ 28

25a. Leaf blades $6-9 \times 2.5-3.5 \mathrm{~cm}$, usually narrowly ovate-lanceolate, acute to slightly acuminate

23. A. padifolia

25b. Leaf blades more than $11 \times 4 \mathrm{~cm}$, usually elliptic to widely ovatelanceolate, longly acuminate

26a. Leaf blades usually bullate; stipules rounded; female bracts lanceolate, entire or subentire

24. A. peruviana

26b. Leaf blades not bullate; stipules lanceolate; female bracts flabellate or triangular-reniform, deeply dentate.

27a. Leaf blade widely ovate-lanceolate, cordate at base

15. A. hibiscifolia

27b. Leaf blade elliptic-lanceolate to oblong-lanceolate, acute at base

19. A. machiensis

28a. Leaf blades linear-lanceolate, no more than $1(-1.5) \mathrm{cm}$ wide

31. A. salicina

28b. Leaf blades not linear-lanceolate, more than $4 \mathrm{~cm}$ wide ............. 29

29a. Leaf blades oblong-lanceolate, pubescent on lower surface, not glossy

37. A. stricta

29b. Leaf blades elliptic-lanceolate or ovate-lanceolate, becoming glabrous, glossy or not ........................................................ 30 30a. Leaf blades ovate-lanceolate, chartaceous; styles unbranched .......... 34. A. simplicistila

30b. Leaf blades elliptic-lanceolate, membranous; styles branched ..... 31

31a. Leaf blades not glossy, margins sparsely toothed; female bracts with linear-lanceolate teeth

36. A. stenoloba

$31 \mathrm{~b}$. Leaf blades usually glossy, margins serrate or crenate-serrate, entire towards the base; female bracts with triangular-lanceolate teeth ....

35. A. stachyura

32a. Leaf blades pinnately (sometimes pinnipalmately) nerved, not cordate at base .......................................................... 33

32b. Leaf blades palmately nerved, more or less cordate at base ........ 38

33a. Leaf blades 8.5-16(-25) cm long ........................................... 34

33b. Leaf blades up to $4.5(-5) \mathrm{cm}$ long ......................................... 35

34a. Leaf blades elliptic-lanceolate or oblong-lanceolate, usually more than $10 \mathrm{~cm}$ long; petioles (0.5-)1-2.5(-5) cm long ....

13. A. diversifolia

34b. Leaf blades narrowly ovate-lanceolate, $8.5-9.5 \mathrm{~cm}$ long; petioles (1.2-) $1.5-2(-2.3) \mathrm{cm}$ long

22. A. neeana

35a. Leaf blades glabrous or nearly so; inflorescences without glandular hairs

35b. Leaf blades pubescent at least at margin and on veins; inflorescences with or without glandular hairs

36a. Leaf blades chartaceous, elliptic or elliptic-lanceolate

4. A. aronioides

36b. Leaf blades thin membranaceous, narrowly lanceolate

18. A. Iycioides

37a. Leaf blades membranaceous, not bullate, glabrous except on veins; inflorescences with glandular hairs ........................... $6 . \boldsymbol{A}$. beckii

37b. Leaf blades coriaceous, bullate, white-tomentose on lower surface; inflorescences without glandular hairs ............. 3. A. argomuelleri

38a. Young branches, leaves and inflorescences usually densely velutinous; glandular hairs absent ................................ 29. A. reflexa

38b. Young branches, leaves and inflorescences not velutinous; glandular hairs present at least in the inflorescences ................................. 39

39a. Young branches, leaves and inflorescences covered with conspicuous glandular hairs ............................................ 26. A. plicata

39b. Glandular hairs present only on inflorescences, sometimes also a few on young branches

40a. Ovaries with glandular hairs

10. A. cuspidata

40b. Ovaries without glandular hairs

2. A. amblyodonta

\section{CATALOGUE TO THE SPECIES OF ACALYPHA OF PERU AND BOLIVIA}

1. Acalypha alopecuroidea Jacq., Collectanea 3: 196 (1789). Ricinocarpus alopecuroides (Jacq.) Kuntze, Revis. Gen. Pl. 2: 617 (1891).

TYPE: [Venezuela] Crescit in Venezuela. Tab. 620 in Jacq., Ic. Pl. Rar. 3 (1792), lectotype, designated by Cardiel (1995[1996]: 233).

Iconography: Jacquin, Icones Plantarum Rariorum 3, Tab. 620. 1792.

Distribution: Southeastern USA, Mexico, Central Ameri$\mathrm{ca}$, Caribbean, northern South America, Brazil and western South America. PERU: San Martín. Not found in Bolivia. We know of only two Peruvian collections, which are from Andean foothills, about $800 \mathrm{~m}$. Disturbed areas (Fig. 1a).

References: Cardiel (2007), Vásquez (2010), Cardiel \& Muñoz (2012a)

The Peruvian collections represent the southern boundary of the known distribution of this species. Macbride (1951) cited this species from Loreto department based on L. Williams 5032, which is $A$. arvensis Poepp.

2. Acalypha amblyodonta (Müll. Arg.) Müll. Arg., Fl. Bras. 
11(2): 365 (1874). Acalypha cuspidata Jacq. var. amblyodonta Müll. Arg. Linnaea 34: 37 (1865). Ricinocarpus amblyodontus (Müll. Arg.) Kuntze, Revis. Gen. Pl. 2: 618 (1891).

TYPE: [Brazil: Rio de Janeiro] In Brasilia prope Rio de Janeiro, C. Gaudichaud 1134 (lectotype, designated here, $\mathrm{P}[\mathrm{P} 00635211]$ !; isolectotypes, $\mathrm{B}$ [presumed destroyed, negative F 5277!], G-DC!. Other type collections: [Brazil] loc. cit., G. Casaretto 1847 (G-DC), A. Weddell 681(G-DC!), J.F. Widgren 190.

Iconography: Lourteig \& O'Donell in Descole, Genera et Species Plantarum Argentinarum, Tab. 93b. 1943.

Distribution: Brazil, western South America (Bolivia and Peru) and southern South America (Paraguay and northern Argentina). BOLIVIA: Santa Cruz (two collections examined). PERU: Cuzco (two collections examined). Andean foothill and Chiquitanía region, 400-1300 m. Dry forests (Fig.1a).

References: Berry (2007), Cardiel (2010).

3. Acalypha argomuelleri Briq., Annuaire Conserv. Jard. Bot. Genève 4: 229 (1900).

TYPE: [Peru: Amazonas] Andes de Chachapoyas, $A$. Mathews s.n. (holotype, G, negative F 24427! ; isotype F[fragment ex G]!).

Acalypha buddleifolia Pax \& K. Hoffm. in Engl., Pflanzenr. 174-16 (Heft 85): 113 (1924).

TYPE: [Peru: Ancash] Huari, unterhalt Chavin de Huantar, in Tale des Puccha-Flusses, $2700 \mathrm{~m}, A$. Weberbauer 3737 (B [presumed destroyed, negative F 5282!]).

Iconography: Unknown.

Distribution: Western South America, Peruvian endemism. PERU: Amazonas, Ancash, Cajamarca and La Libertad (10 collections examined). Not found in Bolivia. Andean region, 2000-2800 m. Shrublands (Fig. 1b).

References: Brako (1993), Cardiel (2007).

Acalypha buddleifolia was described based on the collection A. Weberbauer 3737, from the B herbarium, and presumed destroyed. It would be necessary to designate a neotype.

4. Acalypha aronioides Pax \& K. Hoffm. in Engl., Pflanzenr. 174-16 (Heft 85): 113 (1924).

TYPE: [Peru: Puno] zwischen Sandia und Cuyocuyo, 2100-2200 m, A. Weberbauer 836 (lectotype, designated here, F!; isolectotype B [presumed destroyed, negative $\mathrm{F}$ 5278 !])

Acalypha divaricata Müll. Arg., Linnaea 34:34 (1865), nom. illeg. non Raf. (1836). Ricinocarpus divaricatus (Müll. Arg.) Kuntze, Revis. Gen. Pl. 2: 617 (1891).

TYPE: [Peru] in Peruvia, J.A. Pavón s.n. (holotype, G [negative F 7123!], isotype, MA!, MO!).

Iconography: Unknown.

Distribution: Western South America, Peruvian endemism. PERU: Apurimac, Ayacucho, Cuzco, Huancavelica and Puno (38 collections examined). Not found in Bolivia although expected in La Paz department. Andean region, 2400-4000 m. Rocky slopes (Fig. 1b).

References: Brako (1993), Cardiel (2007).
Saravia (1996) cited Acalypha aronioides from Bolivia based on misidentified specimens of $A$. lycioides Pax \& K. Hoffm. Cardiel (2007) cited this species from Tacna department (Peru) based on herbarium specimen with confused label.

5. Acalypha arvensis Poepp. in Poepp. \& Endl., Nov. Gen. Sp. Pl. 3: 21 (1841). Ricinocarpus arvensis (Poepp.) Kuntze, Revis. Gen. Pl. 2: 617 (1891).

TYPE: [Peru: Amazonas] Crescit in cultis et ruderalis provinciae Maynas ad Yurimaguas, E. Poeppig 2215[2115] (lectotype, W!, designated by Cardiel \& Muñoz (2012: 8); isolectotypes, G [2 sheets]!, F [fragment ex W]!, W!).

Iconography: Dodson \& al. La Flora de Jauneche, Los Ríos. Ecuador: 245, Tab. 92d. 1985.

Distribution: Southeastern U.S.A., Mexico, Central America, Caribbean, northern South America, Brazil and western South America. PERU: Huánuco, Junín, Loreto, Madre de Dios, San Martín and Ucayali (44 collections examined). BOLIVIA: Beni, La Paz and Santa Cruz (29 collections examined). Amazonian region and Andean foothills, 100-600(800) m. Disturbed areas, cultivated fields and shrublands (Fig. 1c).

References: Moraes (1990), Vargas (1993), Cardiel (2007), Cardiel \& Muñoz (2012a).

6. Acalypha beckii Cardiel, Nordic J. Bot. 24(2): 169 (2006).

TYPE: Bolivia: Chuquisaca. Prov. Oropeza de Sucre, pasando Yotala, entrando por la quebrada Nujchu, 2650 m, 15 Oct 1984, S. Beck 8871 (holotype, MA!; isotypes, DAV!, LPB).

Iconography: Cardiel, Nordic Journal of Botany 24(2): 170, fig. 2. 2006.

Distribution: Western South America, Bolivian endemism. BOLIVIA: Chuquisaca. Not found in Peru. We only know two collections, which were made in northern Chuquisaca department. Andean region, $2650 \mathrm{~m}$. Dry forests (Fig. 1d).

References: Cardiel (2006).

7. Acalypha boliviensis Müll. Arg., Linnaea 34: 162 (1865). Ricinocarpus boliviensis (Müll. Arg.) Kuntze, Revis Gen. Pl. 2: 290 (1898).

TYPE: [Bolivia: La Paz] Prov. Larecaja, Sorata, G. Mandon 1070 (holotype, G-DC [G00324655]; isotypes, B [presumed destroyed, negative F 5285!], G, F[2 sheets]!, K!, M!, NY[2 sheets]!, P[3 sheets]!, S[2 sheets]!, W!.

Iconography: Lourteig \& O'Donell in Descole, Genera et Species Plantarum Argentinarum, Tab. 86a. 1943.

Distribution: Western South America (Bolivia) and southern South America (Argentina). BOLIVIA: Chuquisaca, Cochabamba and $\mathrm{La} \mathrm{Paz}$ (4 collections examined). Not found in Peru. Andean region, 1400-2800 m. Rocky slopes (Fig. 1d).

References: Berry (2007).

8. Acalypha communis Müll. Arg., Linnaea 34: 23 (1865). Acalypha communis Müll. Arg. var. tomentosa Müll. Arg., Linnaea 34: 24 (1865). Ricinocarpus communis (Müll. Arg.) Kuntze, Revis. Gen. Pl. 2: 617. 1891.

TYPE: [Brazil: Minas Gerais] In Brasiliae prov. Minarum Generalium, J.F. Widgren s.n. (lectotype, S [S07-12617]! de- 

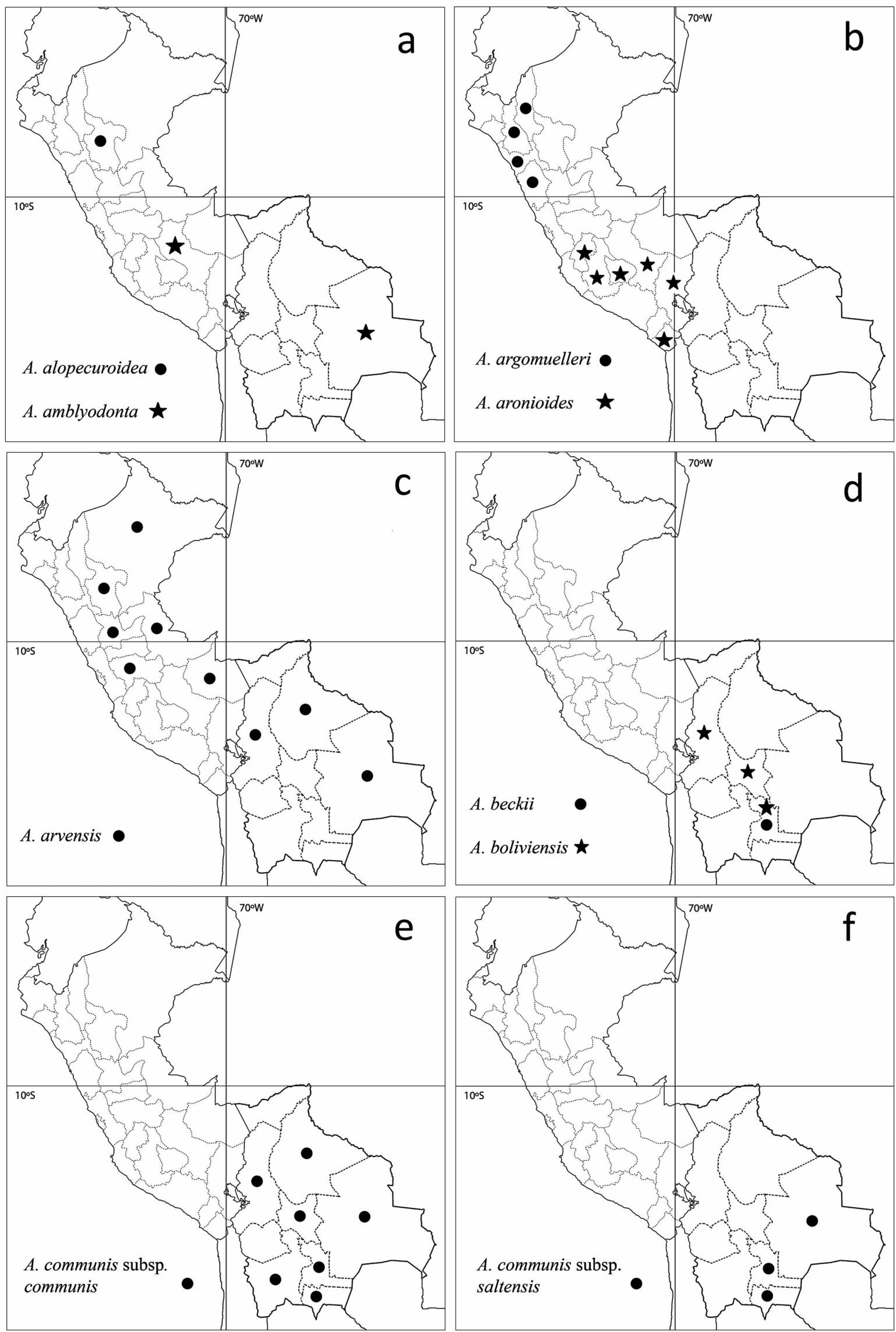

Fig. 1. Maps showing the Bolivian and Peruvian distributions of selected Acalypha species. a, A. alopecuroidea and A. amblyodonta; b, $A$. argomuelleri and $A$. aronioides; $\mathbf{c}, A$. arvensis; $\mathbf{d}, A$. beckii and $A$. boliviensis; $\mathbf{e}, A$. communis subsp. communis; $\mathbf{f}, A$. communis subsp. saltensis. 
signated by Cardiel \& al. (2013: 1300); isolectotype, BR [699 782]).

Iconography: Lourteig \& O'Donell in Descole, Genera et Species Plantarum Argentinarum, Tab. 88b. 1943.

Distribution: Western South America (Bolivia), Brazil, and southern South America. BOLIVIA: Beni, Chuquisaca, Cochabamba, La Paz, Potosí, Santa Cruz and Tarija (57 collections examined). Not found in Peru. Andean and Chiquitanía regions, (300)500-1500(2000) m. Semideciduous forests, Tucumano-Boliviano forests, shrublands and cerrados (Fig. 1e, f).

References: Killeen \& Schulenberg (1998), Serrano \& Terán (1998 [2000]), Arroyo \& Villarroel (2009), Cardiel \& al. (2013).

Acalypha communis belongs to a complex group of species widespread in the northern part of the Southern Cone. This group was reviewed by Cardiel \& al. (2013), who proposed dividing A. communis into five subspecies; two of them are present in Bolivia. Nevertheless we think that hybridization can occur between subspecies because we have observed plants with mixed characters. This group of plants still requires detailed study.

\section{8a. Acalypha communis subsp. communis.}

Distribution: Western South America (Bolivia), Brazil, and southern South America. BOLIVIA: Beni, Chuquisaca, Cochabamba, La Paz, Potosí, Santa Cruz and Tarija (40 collections examined). Not found in Peru. Andean and Chiquitanía regions, 500-1500(2000) m. Semideciduous forest, shrublands and cerrados (Fig. 1e).

References: Cardiel \& al. (2013).

8b. Acalypha communis subsp. saltensis (Pax \& K. Hoffm.) Cardiel \& P. Muñoz, Taxon 62(6): 1301 (2013). Acalypha communis Müll. Arg. var. saltensis Pax \& K. Hoffm. in Engl., Pflanzenr. 147, 16 (heft 85): 39 (1924).

TYPE: [Argentina: Salta] San José, Ufergebüsche, P.G. Lorentz \& Hieronymus 259 (lectotype K!, designated by Cardiel \& al. (2013: 1301).

Iconography: Lourteig \& O'Donell in Descole, Genera et Species Plantarum Argentinarum, Tab. 88a. 1943 [sub A. communis var. saltensis].

Distribution: Western South America (Bolivia) and southern South America (northern Argentina). BOLIVIA: Chuquisaca, Santa Cruz and Tarija (17 collections examined). Not found in Peru. Andean foothills and Chiquitanía region, (300)500-1000(1400) m. Dry forests and Tucumano-Boliviano forests (Fig. 1f).

References: Cardiel \& al. (2013).

This subspecies has a well-defined distribution along the eastern Andean slopes in southern Bolivia and northern Argentina that seems to be associated with the Tucumano-Boliviano forest. Some Santa Cruz collections show intermediate characters with $A$. communis subsp. communis.

9. Acalypha cuneata Poepp. in Poepp. \& Endl., Nov. Gen. Sp. Pl. 3: 22 (1845). Ricinocarpus cuneatus (Poepp.) Kuntze, Revis. Gen. Pl. 2: 617 (1891). Acalypha obovata Benth. var. cuneata (Poepp.) J.F. Macbr., Candollea 8: 26 (1940).
TYPE: [Peru: Amazonas] Crescit in fruticetis maynensibus ad Yurimaguas. Martio lecta, E. Poeppig 2230 (lectotype, W[113778]!, designated by Cardiel \& Muñoz (2012a), isolectotypes, $\mathrm{B}$ [presumed destroyed, negative F 5288], F!, W!). Other type collections: [Peru: Amazonas] loc. cit., E. Poeppig 2317 (B[presumed destroyed, negative 5288], F!, G!, $\mathrm{P}[2$ sheets]!, W!), 2330 (F!, G[4 sheets]!, P[2 sheets]!, W!), 2807 (W!).

Acalypha erosa Rusby, Bull. Torrey Bot. Club 28: 305 (1901).

TYPE: [Bolivia: La Paz] Prov. Larecaja, Guanai, $2000 \mathrm{ft}$, May 1886, H.H. Rusby 1757 (lectotype, designated here, NY[246106]!; isotype, NY[312664]!).

Iconography: Bentham, The Botany of the voyage of H.M.S. Sulphur, Tab. 53.1844 [sub. A. obovata].

Distribution: Central America (Panama), northern South America, Brazil, western South America. PERU: Amazonas, Cuzco, Huánuco, Junín, Loreto, Madre de Dios, Pasco and San Martín (124 collections examined). BOLIVIA: Beni, La Paz and Santa Cruz (15 collections examined). Amazonian region and Andean foothills, 100-1000(1900) m. Seasonally inundated rain forests and cloud forests (Fig. 2a).

References: Parker \& Bailey (1991), Vargas (1993), Brako (1993), Cardiel (2007), Berry (2007), Vásquez (1997, 2010), Cardiel \& Muñoz (2012a).

10. Acalypha cuspidata Jacq., Pl. Hort. Schoenbr. 2: 63, Tab. 243 (1797). Ricinocarpus cuspidatus (Jacq.) Kuntze, Revis. Gen. Pl. 2: 617 (1891).

TYPE: [Venezuela] Crescit ad Caracas, Tab. 243 in Jacq., loc. cit. (1797), lectotype designated by Cardiel (1995[1996]: 233).

Iconography: Jacquin, Plantarum rariorum horti caesarei schoenbrunnensis, Tab. 243. 1797.

Distribution: Mexico, Caribbean, northern South America, western South America. PERU: Tumbes. Not found in Bolivia. We know of a single collection. Coastal region, 700-800 m. Deciduous forests (Fig. 2a).

References: Berry (2007), Cardiel (2007), Cardiel \& Muñoz (2012a).

The Peruvian collection represents the southern boundary of the known distribution of this species. Foster (1958) cited this species from Bolivia based on misidentified specimens of A. villosa Jacq.

11. Acalypha delicata Cardiel, Nordic J. Bot. 24(2): 167 (2006).

TYPE: Peru. Province of Tumbes. Mts. E. of Hacienda Chicama, 800-900 m, 19-24 Feb 1927, A. Weberbauer 7641 (holotype, F!; isotype, NY!).

Iconography: Cardiel, Nordic Journal of Botany 24(2): 168, fig. 1. 2006.

Distribution: Western South America, Peruvian endemism. PERU: Tumbes. We know of a single collection, from southern Tumbes department. Coastal region, 800-900 m. Deciduous brushwoods (Fig. 2b).

References: Cardiel (2007). 

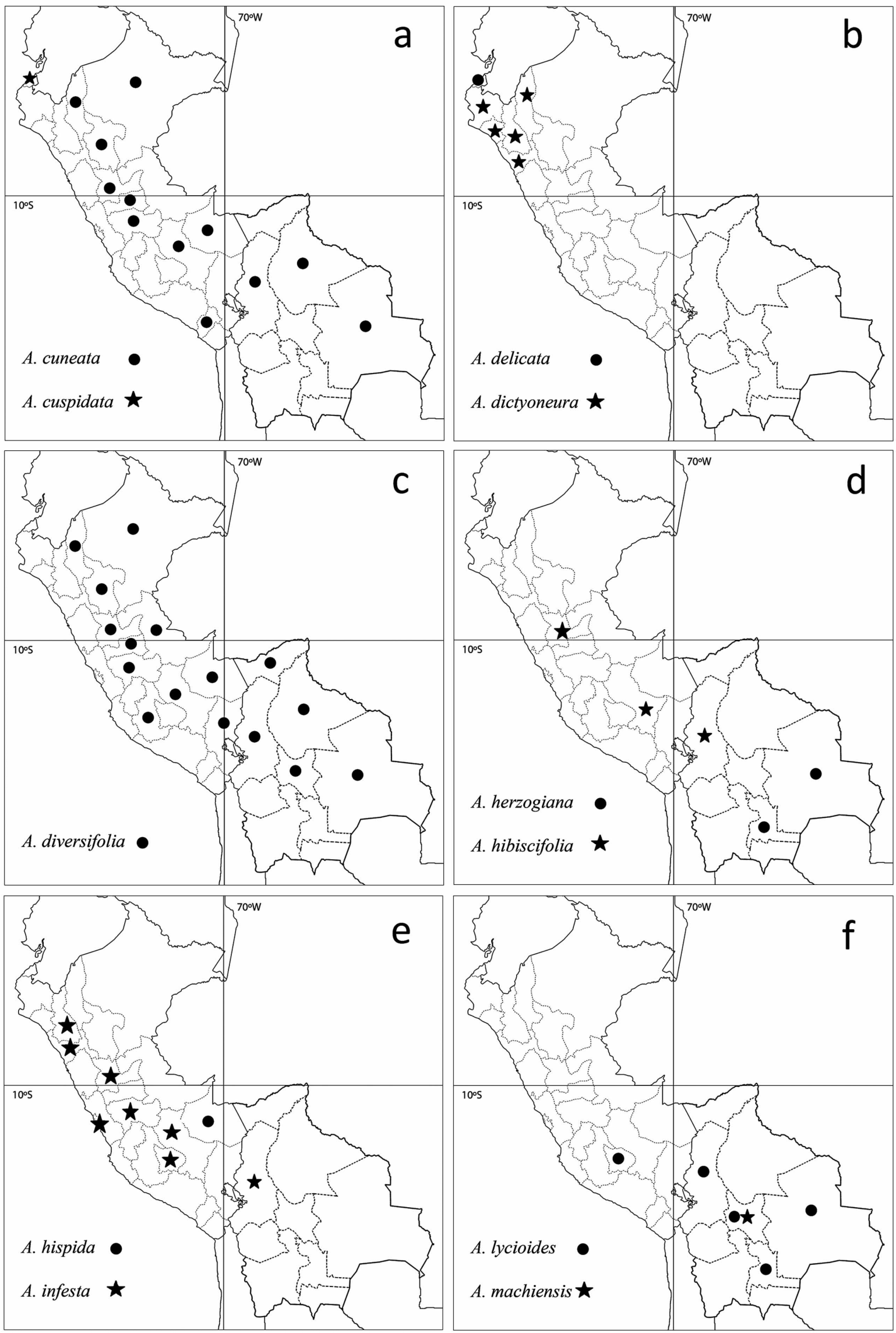

Fig. 2. Maps showing the Bolivian and Peruvian distributions of selected Acalypha species. a, A. cuneata and A. cuspidata; b, A. delicata and A. dictyoneura; c, A. diversifolia; d, A. herzogiana and A. hibiscifolia; e, A. hispida and A. infesta; f, A. lycioides and A. machiensis. 
12. Acalypha dictyoneura Müll. Arg., Linnaea 34: 12 (1865). Ricinocarpus dictyoneurus (Müll. Arg.) Kuntze, Revis Gen. Pl. 2: 617 (1891).

TYPE: [Peru: Amazonas] In Peruvia prope Chachapoyas, A. Matthews s.n. (holotype, G!; isotype, $\mathrm{K}$ !).

Acalypha dictyoneura Müll. Arg. f. reducta Müll. Arg., Linnaea 34: 13 (1865). Acalypha dictyoneura Müll. Arg. var. reducta (Müll. Arg.) J.F. Macbr., Fieldiana, Bot. 13(3a/1): 129 (1951).

TYPE: [Peru] In Peruvia, J.A. Pavón s.n. (lectotype, designated here, G-DC [G00324032]!; isolectotype B [presumed destroyed, negative F 5298!]).

Iconography: Unknown.

Distribution: Western South America (Ecuador and Peru). PERU: Amazonas, Cajamarca, La Libertad, Lambayeque and Piura (20 collections examined). Not found in Bolivia. Andean region, 1500-2500 m. Cloud forests (Fig. 2b).

References: Brako (1993), Cardiel (2007), Cardiel \& Muñoz (2012a).

13. Acalypha diversifolia Jacq., P1. Hort. Schoenbr. 2: 63, Tab. 244 (1797). Acalypha leptostachya Kunth $\mathrm{f}$. diversifolia (Jacq.) Müll. Arg., Linnaea 34: 35. 1865. Ricinocarpus diversifolius (Jacq.) Kuntze, Revis. Gen. Pl. 2: 617. 1891.

TYPE: [Venezuela] ex Caracas, Tab. 244 in Jacq., Pl. Hort. Schoenbr. 2 (1797), lectotype designated by Cardiel (1995[1996]: 233).

Acalypha samydifolia [samydaefolia] Poepp. in Poepp. \& Endl., Nov. Gen. Sp. Pl. 3: 21, Tab. 224 (1844). Ricinocarpus samydifolius (Poepp.) Kuntze, Revis. Gen. Pl. 2: 648 (1894).

TYPE: [Peru: Amazonas] crescit in sylvis maynensibus ad Yurimaguas, E. Poeppig 2122 (lectotype, designated here, $\mathrm{W} !$, negative 2496; isolectotypes, B [presumed destroyed, negative F 5138!], G[2 sheets]!, P[2sheets]!,W!).

Acalypha microgyna [microgyne] Poepp. in Poepp. \& Endl., Nov. Gen. Sp. Pl. 3: 21 (1844).

TYPE: [Peru: Huánuco] Crescit in sylvis Peruviae transandinis ad Cuchero. Not located.

Acalypha leptostachya Kunth var. carpinifolia Poepp. ex Müll.

Arg., Linnaea 34: 35 (1865). Acalypha diversifolia Jacq. var. carpinifolia (Poepp. ex Müll.Arg.) Müll. Arg. in DC., Prodr. 15(2): 854 (1866).

TYPE: [Peru] in Peruvia, E. Poeppig 1307 (lectotype, designated here, W!; isolectotypes, F!, P!, US!). Other type collections: [Colombia] In Nova Granata, Balbis s.n., [Venezuela] in Venezuela, A. Fendler 1261 (K!), [Mexico] in Mexico, J. Linden 5.

Acalypha inaequalis Rusby, Bull Torrey Bot. Club 28: 303 (1901).

TYPE: [Bolivia: La Paz] Yungas, $6000 \mathrm{ft}, 1885$, M. Bang 1947 (lectotype, designated here, NY [246116]!; isolectotypes, NY!, F!, US!).

Acalypha alchorneoides Rusby, Bull. New York Bot. Gard. 8(28): 101 (1912).
TYPE: [Bolivia: La Paz] Prov. Iturralde, San Buena Ventura, $1400 \mathrm{ft}, 12$ Nov. 1901, R.S. Williams 674 (lectotype, designated here, NY[246089]!; isolectotypes, NY!, K!).

Acalypha vermifera Rusby, Mem. New York Bot. Gard. 7: 286. 1927.

TYPE: [Bolivia: Beni] Huachi, $1800 \mathrm{ft} 2 \mathrm{Sep}$ 1921, E.O. White 944 (lectotype, designated here, NY[312929]!; isolectotypes, MICH!, NY!).

Iconography: Jacquin, Plantarum rariorum horti caesarei schoenbrunnensis, Tab. 244. 1797.

Distribution: Mexico, Central America, northern South America, Brazil, western South America. PERU: Amazonas, Ayacucho, Cuzco, Huánuco, Junín, Loreto, Madre de Dios, Pasco, Puno, San Martín and Ucayali (150 collections examined). BOLIVIA: Beni, Cochabamba, La Paz, Pando and Santa Cruz (52 collections examined). Amazonian and Andean region, 100-1000(2000) $\mathrm{m}$. Rain forests to disturbed areas, usually along streams (Fig. 2c).

References: Parker \& Bailey (1991), Vargas (1993), Brako (1993), Killeen \& al. (1993), Alverson \& al. (2000), Cardiel (2007), Valenzuela \& al. (2007), Vásquez (2010), Cardiel \& Muñoz (2012a).

14. Acalypha herzogiana Pax \& K. Hoffm., Meded. RijksHerb. 40: 24 (1921).

TYPE: [Bolivia: Santa Cruz] in Walde zwischen Rio Pirai und Rio Cuchi, 450 m, T. Herzog 1453 (lectotype, designated here, S[S-R-7754]!; isolectotypes, B[presumed destroyed, negative F 5294!], Z!).

Iconography: Unknown.

Distribution: Western South America (Bolivia), Brazil, southern South America (Paraguay). BOLIVIA: Chuquisaca and Santa Cruz (17 collections examined). Not found in Peru. Amazonian and Chiquitanía region, 300-500 m. Dry forests and open sandy areas (Fig. 2d).

References: Foster (1958), Steinmann \& Levin (2011).

15. Acalypha hibiscifolia Britton ex Rusby, Mem. Torrey Bot. Club 4(3): 257 (1895).

TYPE: [Bolivia: La Paz] Yungas, H.H. Rusby 1275 (lectotype, designated here, NY [246115]!; isolectotype NY!).

Acalypha buchtienii Pax, Repert. Spec. Nov. Regni Veg. 5: 227 (1908).

TYPE: [Bolivia: La Paz] Sud Yungas, Yanacachi, $1700 \mathrm{~m}$, 16 Jun 1906., O. Buchtien 377 (lectotype, designated here, US [1158292]!; isolectotype B [presumed destroyed]).

Iconography: Fig. 3.

Distribution: Western South America (Bolivia and Peru). PERU: Cuzco and Huánuco (2 collections examined). BOLIVIA: La Paz (33 collections examined). Andean region, [500]800-2000 m. Cloud forests (Fig. 2d).

References: Berry (2007), Cardiel (2007).

16. Acalypha hispida Burm. f., Fl. Ind. 302, Tab. 61, f. 1 (1768). Ricinocarpus hispidus (Burm. f.) Kuntze, Revis. Gen. Pl. 2: 618 (1891).

TYPE: Habitat in India, Tab. 61 in Burm. f., loc. cit. 302 (1768). 


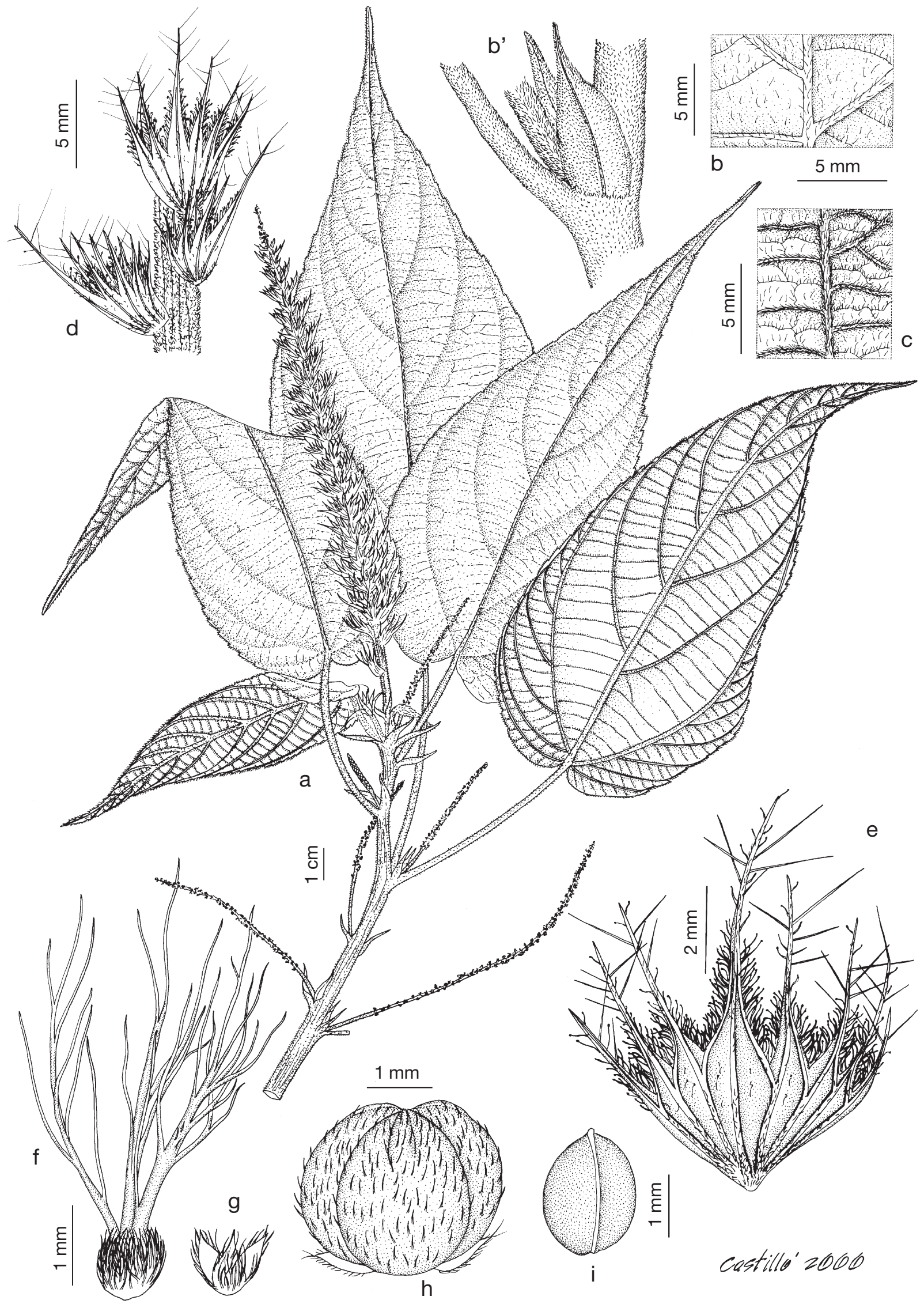

Fig. 3. Acalypha hibiscifolia. $\mathbf{a}$, flowering branch; $\mathbf{b}$, detail of the leaf upper surface; $\mathbf{b}^{\mathbf{2}}$, stipules; $\mathbf{c}$, detail of the leaf lower surface; $\mathbf{d}$, detail of the female inflorescence; e, female developed bract; $\mathbf{f}$, ovary and styles; $\mathbf{g}$, calyx of female flower; $\mathbf{h}$, capsule; i, seed. a-g, J.C. Solomon 8530 (F, MO); h, i, J.C. Solomon \& M. Nee 14271 (MO). 
Iconography: Burmann, Flora indica, Tab. 61, fig., 1. 1768.

Distribution: Native to Malaysia or Melanesia; grown in gardens throughout the tropics and frequently appearing naturalized despite not producing seeds. PERU: Madre de Dios (one collection examined). Not found in Bolivia but expected there. Amazonian region, $200 \mathrm{~m}$. Urban areas (Fig. 2e).

References: Brako (1993), Cardiel (2007), Cardiel \& Muñoz (2012a).

17. Acalypha infesta Poepp. in Poepp. \& Endl., Nov. Gen. Sp. Pl. 3: 22 (1845). Ricinocarpus infestus (Poepp.) Kuntze, Revis. Gen. Pl. 2: 617 (1891). Acalypha infestans Müll. Arg. var. stenoloba Müll. Arg., Linnaea 34: 23 (1865).

TYPE: [PERU: Huánuco] Crescit in cultis ad Cuchero, E. Poeppig 1701 (lectotype, W [103476]!, designated by Cardiel \& Muñoz (2012a: 10); isolectotypes, F!, P!, US!, W!).

Acalypha infesta var. rotundifolia Müll. Arg., Linnaea 34: 23 (1865).

TYPE: [Peru] In Peruvia, J. Dombey s.n. (lectotype, designated here, $\mathrm{P}$ [P00635275]!; isolectotypes, P [2 sheets]!. Other type collection: [Peru] In Peruvia, J.A. Pavón 36 (P!)

Acalypha forbesii S. Moore, J. Bot. 336: 52 (1914).

TYPE: [Peru: Lambayeque] in valley between Pascamayo and Railhead, $7000 \mathrm{ft}$, H.O. Forbes s.n. (holotype, BM!).

Iconography: Cardiel, Revista de la Academia Colombiana de Ciencias Exactas, Físicas y Naturales 97: 464, fig. 1. 2001.

Distribution: Western South America. PERU: Apurimac, Cajamarca, Cuzco, Huánuco, Junín, La Libertad and Lima (39 collections examined). BOLIVIA: La Paz (2 collections examined). Coastal and Andean regions, 100-2500(2800) m. Shrublands and cultivated fields (Fig. 2e).

References: Brako (1993), León \& al. (2006), Cardiel (2007), Cardiel \& Muñoz (2012a).

Acalypha infesta is rarely cited or collected, and often is confused with $A$. poiretii Spreng.

18. Acalypha lycioides Pax \& K. Hoffm., Meded. Rijks Herb. 40: 24 (1921).

TYPE: [Bolivia: Santa Cruz] Bergwald bei Charagua, 1000 m, T. Herzog 1213 (lectotype, designated here, Z [15838]!; isolectotypes, S!, F!, Z[15839]!).

Iconography: Lourteig \& O'Donell in Descole, Genera et Species Plantarum Argentinarum, Tab. 91. 1943.

Distribution: Western South America (Peru and Bolivia) and southern South America (Argentina). PERU: Apurimac (1 collection examined). BOLIVIA: Chuquisaca, Cochabamba, La Paz and Santa Cruz (30 collections examined). Andean region, [500]1000-2500 m. Dry forests, shrublands, and rocky slopes (Fig. 2f).

References: Serrano \& Terán (1988 [2000]), Berry (2007).

19. Acalypha machiensis Cardiel \& $\mathrm{P}$. Muñoz, Brittonia 64(4): 365 (2012).

TYPE: Bolivia: Cochabamba. Province of Chapare, Villa Tunary, pasando el río, Parque Machía. $17^{\circ} 03^{\prime} \mathrm{S} 65^{\circ} 29^{\prime} \mathrm{W}$, 479 m, 22 Sep 2002, S. Beck 28576 (holotype, LPB!; isotype, MA!).
Iconography: Cardiel \& Muñoz, Brittonia 64(4): 366, fig. 2. 2012.

Distribution: Western South America, Bolivian endemism. BOLIVIA: Cochabamba. We only know one collection, made at the foot of the eastern slope of the Cordillera Oriental, in Cochabamba department, at $469 \mathrm{~m}$. Andean foothill. Rain forests (Fig. 2f).

References: Cardiel \& Muñoz (2012b).

20. Acalypha macrostachya Jacq., Pl. Hort. Schoenbr. 2: 63, t. 245 (1797). Ricinocarpus macrostachyus (Jacq.) Kuntze, Revis. Gen. Pl. 2: 618 (1891).

TYPE: [Venezuela] Crescit ad Caracas (lectotype, Tab. 245 in Jacq., Pl. Hort. Schoenbr. 2. 1797, designated by Cardiel, 1995[1996]: 233).

Acalypha tristis Poepp. in Poepp. \& Endl., Nov. Gen. Sp. Pl. 3: 22 (1845). Acalypha macrostachya Jacq. var. tristis (Poepp.) Müll. Arg. in Mart., Fl. Bras. 11(2): 345 (1874).

TYPE: [Peru: Loreto] Crescit in fruticetis maynensibus ad Yurimaguas, E. Poeppig 3088 (lectotype, designated here, G!; isolectotypes, G!, W, F [fragment ex W]!)

Acalypha tarapotensis Müll. Arg. in DC., Prodr. 15(2): 808 (1866). Ricinocarpus tarapotensis (Müll. Arg.) Kuntze, Revis. Gen. Pl. 2: 618 (1891).

TYPE: [Peru: San Martín] In Peruvia orientali prope Tarapoto, R. Spruce 4298 (holotype, G-DC [G00324051]; isotypes, BM!, G [2 sheets]!, GH!, K [2 sheets]!, NY!, W!).

Acalypha foliosa Rusby, Mem. Bull. New York Bot. Gard. 4(14): 443 (1907).

TYPE: [Bolivia: La Paz] Nor Yungas, Coroico, 20 Aug 1894, M. Bang 2391 (lectotype, designated here, NY [246109]!; isolectotypes, BM!, C!, F[2 sheets]!, G!, K!, M!, MA!, MICH!, MO!, NY[3 sheets]!, US[2 sheets]!, W!, Z[2 sheets]!).

Acalypha williamsii Rusby, Bull. New York Bot. Gard. 8(28): 101 (1912). Non Rusby (1920).

TYPE: [Bolivia: La Paz] Prov. Iturralde, San Buena Ventura, 1500 ft, 20 Nov 1901, R.S. Williams 656 (lectotype, designated here, NY [246144]!; isolectotypes, MA!, NY!, K! US!).

Acalypha beteromorpha Rusby, Mem. New York Bot. Gard. 7: 286 (1927).

TYPE: [Bolivia, Beni] Prov. Ballivián, Rubenabaque, 1000 ft, 7 Oct 1921, M. Cárdenas 1554 (lectotype, designated here, NY [312930]!; isolectotype, NY!).

Iconography: Jacquin, Plantarum rariorum horti caesarei schoenbrunnensis, Tab. 245. 1797.

Distribution: Mexico, Central America, Caribbean (Grenada), northern South America, Brazil, western South America. PERU: Amazonas, Ayacucho, Cajamarca, Cuzco, Huánuco, Junín, Loreto, Madre de Dios, Pasco, San Martín and Ucayali (84 collections examined). BOLIVIA: Beni, Cochabamba, La Paz, Pando and Santa Cruz (55 collections examined). Amazonian and Andean regions, 100-2500 m. Rain forest, montane forest, shrublands and disturbed areas (Fig. 4a). 

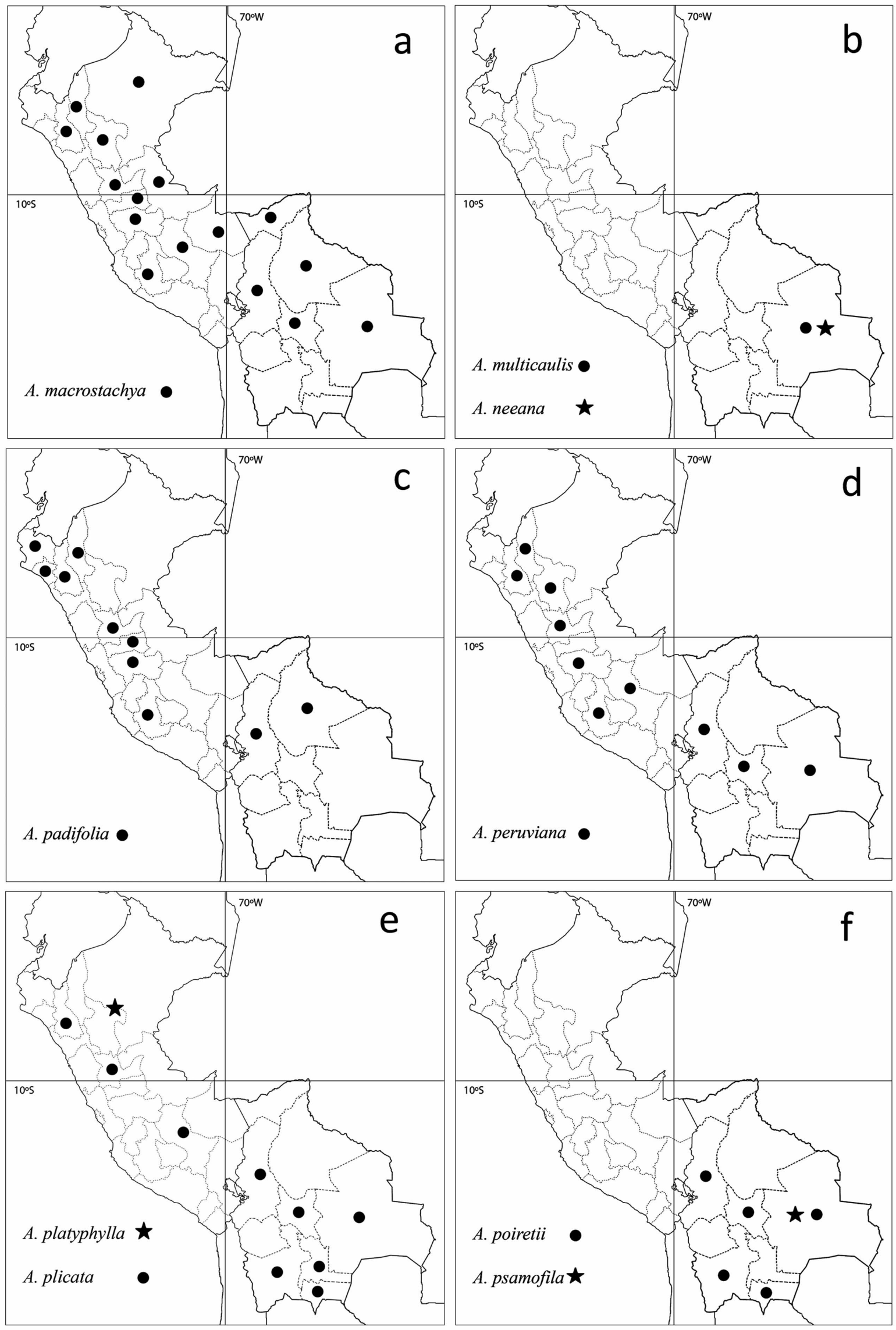

Fig. 4. Maps showing the Bolivian and Peruvian distributions of selected Acalypha species. a, $A$. macrostachya; b, $A$. multicaulis and $A$. neeana; $\mathbf{c}$ A. padifolia; d, A. peruviana; e, A. platyphylla and A. plicata; f, A. poiretii and A. psamofila. 
References: Parker \& Bailey (1991), Vargas (1993), Killeen \& Schulenberg (1998), Pennington \& al. (2004), Berry (2007), Cardiel (2007), Valenzuela \& al. (2007), Vásquez $(1997,2010)$, Cardiel \& Muñoz (2012a).

21. Acalypha multicaulis Müll. Arg., Linnaea 34: 53 (1865). Ricinocarpus multicaulis (Müll. Arg.) Kuntze, Revis. Gen. Pl. 2: 618 (1891).

TYPE: [Brasil] In Brasilia, F. Sellow s.n. (neotype, designated here, $\mathrm{K}$ !).

Iconography: Lourteig \& O'Donell in Descole, Genera et Species Plantarum Argentinarum, Tab. 92a. 1943.

Distribution: Western South America (Bolivia) and southern South America (Paraguay, Uruguay and Argentina). BOLIVIA: Santa Cruz (2 collections examined). Not found in Peru. Chiquitanía region, $400 \mathrm{~m}$. Dry forests (Fig. 4b).

References: Berry (2007), Arroyo \& Villarroel (2009).

Acalypha multicaulis was described based on a single collection, F. Sellow s.n. from the B herbarium, presumed destroyed; a negative made by Francis Macbride is preserved (negative F 5304!). Because we located no duplicates, we designate as neotype another Sellow's collection of this species from Brazil, found in the $\mathrm{K}$ herbarium.

22. Acalypha neeana Cardiel \& P. Muñoz, Brittonia 64(4): 363 (2012).

TYPE: Bolivia: Santa Cruz. Province of Florida, $2.5 \mathrm{~km}$ NE of main highway at Mairana, Quebrada Yesera, M. Nee $\mathcal{E}$ I. Vargas 44687 (holotype, LPB!; isotypes, MA!, MO!, NY!, USZ!).

Iconography: Cardiel \& Muñoz, Brittonia 64(4): 364, fig. 1. 2012.

Distribution: Western South America, Bolivian endemism. BOLIVIA: Santa Cruz. Not found in Peru. We only know one collection, made on the eastern slopes of the Cordillera Oriental, in Santa Cruz department. Andean region, 1550 m. Deciduous forests (Fig. 4b).

References: Cardiel \& Muñoz (2012b).

23. Acalypha padifolia Kunth in Humb. \& Bonpl., Nov. Gen. Sp. (quarto ed.) 2: 97 (1817). Ricinocarpus padifolius (Kunth) Kuntze, Revis. Gen. Pl. 2: 618 (1891).

TYPE: [Colombia: Nariño] Crescit locis sylvaticis subfrigidis inter Almaguer et Pasto, prope villam Meneses, alt. 1322 hex, A. Humboldt \& A. Bonpland 2136 (holotype, P-Bonpl.!; isotype, $\mathrm{P}$ !).

Acalypha macrodonta Müll. Arg., Linnaea 34: 51 (1865). Ricinocarpus macrodontus (Müll. Arg.) Kuntze Revis. Gen. Pl. 2: 618 (1891).

TYPE: [Peru] In Peruvia, J.A. Pavón s.n. (holotype, G-DC [G00324843], negative F 8501!).

Acalypha ruiziana Müll. Arg., Linnaea 34: 16 (1865).

TYPE: [Peru] In Peruvia, J.A. Pavón s.n. (lectotype, designated here, MA [812800]!; isolectotype, MA [812799]!). Other type collection: [Peru] loc. cit., H. Ruiz s.n., B [presumed destroyed, negative F 5316!], K!).

Iconography: Unknown.
Distribution: Western South America. PERU: Amazonas, Ayacucho, Cajamarca, Huánuco, Junín, Lambayeque, Pasco and Piura (45 collections examined). BOLIVIA: Beni and La $\mathrm{Paz}$ (11 collections examined). Andean region, [800]1200$2000 \mathrm{~m}$. Forests, shrublands and disturbed areas (Fig. 4c).

References: Vargas (1993), Brako (1993), Cardiel (2007), Cardiel \& Muñoz (2012a).

24. Acalypha peruviana Müll. Arg., Linnaea 34: 17 (1865). Ricinocarpus perwvianus (Müll. Arg.) Kuntze, Revis. Gen. Pl. 2: 618 (1891).

TYPE: [Peru] In Peruvia, H. Ruiz s.n. (lectotype, designated here, K [K000600514]!; isolectotype, B [presumed destroyed, negative F 5308!]. Other type collection: J.A. Pavón s.n. (G-DC, MA!).

Acalypha bullata Müll. Arg., Linnaea 34: 17 (1865).

TYPE: [Peru] In Peruvia, H. Ruiz s.n. (holotype, G-DC [G00324366]; isotype, B [presumed destroyed, negative F $5283 !])$.

Acalypha subbullata Pax \& K. Hoffm. in Engl., Pflanzenr. 147-16(85): 67 (1924).

TYPE: [Peru: Junín] Pariahuanca. A. Mathews 1199 (holotype, W!, negative 32507).

Ricinocarpus controversus Kuntze, Revis. Gen. Pl. 3(2): 290 (1898). Acalypha controversa (Kuntze) K. Schum., Just. Bot. Jahresber. 26(1): 348 (1900).

TYPE: [Bolivia: Cochabamba] Río Juntas, $1600 \mathrm{~m}, O$. Kuntze s.n. (lectotype, designated here, NY [273203]!; isolectotype, NY!).

Iconography: Unknown.

Distribution: Western South America (Peru and Bolivia). PERU: Amazonas, Ayacucho, Cajamarca, Cuzco, Huánuco, Junín and San Martín (28 collections examined). BOLIVIA: Cochabamba, La Paz and Santa Cruz 5 collections examined). Andean region, 1500-3150 m. Cloud forests (Fig. 4d).

References: Brako (1993), Vargas (1993), León \& al. (2006), Cardiel (2007).

25. Acalypha platyphylla Müll. Arg., Linnaea 34: 6 (1865). Ricinocarpus platyphyllus (Müll. Arg.) Kuntze, Revis. Gen. Pl. 2: 618 (1891).

TYPE: [Ecuador] in Ecuador peruviae, L. Fraser, s.n. (holotype, G-DC 324093!, isotype BM!).

Acalypha subandina Ule, Verh. Bot. Vereins Prov. Brandenburg 50: 77 (1908).

TYPE: [Peru: currently department of San Martín] Departamento de Loreto, Cerro de Escalero, 1200 m, E. Ule 6840 (lectotype, G! designated by Muñoz-Rodríguez \& al. (2014: 211); isolectotypes, B [presumed destroyed, negative F 5322!], CORD!, K!, MG!).

Iconography: Cardiel, Acalypha in Flora de Colombia 15: 48, fig. 13. 1995 .

Distribution: Western South America (Colombia, Ecuador and Peru). PERU: San Martín (one collection examined). Not 
found in Bolivia. We only know the type collection of $A$. subandina. Andean foothills, $1200 \mathrm{~m}$. Montane forests (Fig. $4 \mathrm{e})$.

References: Brako (1993), Cardiel (2007), Cardiel \& Muñoz (2012a), Muñoz-Rodríguez \& al. (2014).

Acalypha platyphylla is widely distributed in the Colombian and Ecuadorian Andes. The Peruvian specimen points the southern boundary in the known distribution of this species. Ulloa Ulloa \& al. (2004) cited another collection from Peru, identificated as A. subandina, based on Schunke 8458 (MO), which is of $A$. stenoloba.

26. Acalypha plicata Müll. Arg. in DC., Prodr. 15(2): 855 (1866). Ricinocarpus plicatus (Müll. Arg.) Kuntze, Revis. Gen. Pl. 2: 618 (1891).

TYPE: [Bolivia] In Bolivia, T. Bridges s.n. (holotype, GDC [G00324504]!, negative F 7124]; isotype K!).

Acalypha flabellifera Rusby, Mem. Torrey Bot. Club 6: 119 (1896).

TYPE: [Bolivia: Cochabamba] Near snow line, Mt. Tunari (?). M. Bang 1109 (lectotype, designated here, NY [00246108]!; isolectotypes, BM!, F!, G!, GH[2 sheets]!, K!, MA!, MO!, NY[3 sheets]!, US[2 sheets]!).

Acalypha fulva I.M. Johnst., Contr. Gray Herb. 75: 29 (1925).

TYPE: [PERU: Huánuco] Muña, 2100 m, J.F. Macbride 4011 (holotype, F [535075]!, negative 50138; isotypes, GH, US!).

Ricinocarpus cuspidatus (Jacq.) Kuntze var. glandulosus Kuntze, Revis. Gen. Pl. 3(2): 291 (1898).

TYPE: [Bolivia: Cochabamba] Tunarigebirge, $3000 \mathrm{~m}, O$. Kuntze s.n. (holotype, NY[273204]!).

Iconography: Lourteig \& O'Donell in Descole, Genera et Species Plantarum Argentinarum, Tab. 90. 1943 [sub. A. flabellifera].

Distribution: Northern South America, western South America and southern South America. PERU: Cajamarca, Cuzco and Huánuco ( 5 collections examined). BOLIVIA: Cochabamba, Chuquisaca, La Paz, Potosí, Santa Cruz and Tarija (60 collections examined). Andean region, (1200)15003000(3600) m. Dry forests, shrublands, rocky slopes and sandy places (Fig. 4e).

References: Vargas (1993), Serrano \& Terán (1998[2000]), Cardiel (2007), Berry (2007).

The type locality of Acalypha flabellifera "near snowline Mt. Tunari" is only penciled onto one sheet (NY 246108); this and other collections with this locality are undoubtedly in error and were collected much further down the Andean slopes to the north. Bang's notebooks from these Cochabamba collections were lost, resulting in very imprecise locality data.

27. Acalypha poiretii Spreng., Syst. Veg. 3: 879 (1826). Ricinocarpus poiretii (Spreng.) Kuntze, Revis. Gen. Pl. 3(2): 618 (1891).

TYPE: “Amer. trop.”, Anonymous s.n. (holotype, P-LAM!).

Acalypha paupercula Pax \& K. Hoffm., Meded. Rijks-Herbar. 40: 24. 1921. syn. nov.
TYPE: [Bolivia. Santa Cruz] Prov. Cordillera, Camatindi, $700 \mathrm{~m}$, T. Herzog 1167 (lectotype, designated here, S [S-R 7739]!; isolectotype, Z!).

Iconography: Lourteig \& O'Donell in Descole, Genera et Species Plantarum Argentinarum, t. 86b. 1943.

Distribution: South-central USA., Mexico, Central America, Caribbean, northern South America, Brazil, western South America (Bolivia), southern South America (Argentina). BOLIVIA: Cochabamba, La Paz, Potosí, Santa Cruz and Tarija ( 9 collections examined). Not found in Peru. Andean region, 800-2300 m. Scrublands and disturbed areas (Fig. 4f).

References: Berry (2007), Cardiel (2007), Guantay \& al. (2008).

Although poorly developed, Acalypha paupercula appears to match $A$. poiretii and therefore we propose treating it as a synonmy of $A$. poiretii.

\section{Acalypha psamofila sp. nov.}

TYPE: Bolivia: Santa Cruz. Prov. Andrés Ibáñez: Along highway from Santa Cruz to Abapó, $3 \mathrm{~km} \mathrm{~S}$ of crossing of railroad and $2 \mathrm{~km} \mathrm{~S}$ of bridge over Quebrada Pejí, 17 ${ }^{\circ} 58^{\prime} \mathrm{S}$, $63^{\circ} 11^{\prime} \mathrm{W}, 450$ m, 27 Dec 1988, M. Nee, G. Coimbra, A. Araujo $\mathcal{E} S$. Nogales 48480 (holotype, MA [630580]!; isotypes, $\mathrm{LPB}, \mathrm{NY}$ ).

Species Acalyphae vellameae Baill. similis, a qua praecipue differt petiolis longioribus (usque 4,5 $\mathrm{cm}$ vs usque 1,5 cm in A. vellamea) atque stipulis inconspicuis (vs usque $13 \mathrm{~mm}$ in A. vellamea).

\section{Iconography: Fig. 5.}

Shrubs or subshrubs c. $0.5 \mathrm{~m}$ high, monoecious; young branches thick, divaricate, densely velutinous-pubescent, with simple, slender hairs; older branches glabrescent. Stipules inconspicuous, linear-lanceolate, to $2.5 \mathrm{~mm}$ long, subacute, hispidulous, caducous. Petiole (1.5)2-3.5(5) cm long, with indumentum similar to that found on the young branches. Leaf blade 3.5-5(7) $\times 3-4(6) \mathrm{cm}$, broadly ovate to subtriangular, membranaceous; the base rounded to truncate; the apex subobtuse to apiculate; the margin crenate-dentate, teeth rounded to subacute; upper surface pubescent with simple, thin hairs; lower surface densely pubescent, subvelutinous with simple, whitish hairs, with sparse bright yellow minute droplets; venation palmate to pinnipalmate, with 4-5 veins per side. Stipels absent. Inflorescences unisexual. Male inflorescences axillary, 3-4(5) cm long, spicate; peduncle 1-3 $\mathrm{cm}$ long, thick, densely pubescent; rachis hispidulous; flowers glomerate; bracts minute, to $1 \mathrm{~mm}$ long, linear-lanceolate, ciliate-hispidulous. Female inflorescences numerous, terminal and axillary, erect, to $8(10) \mathrm{cm}$ long; peduncle $1.5-2 \mathrm{~cm}$ long, thick, with indumentum similar to that found on the young branches; rachis densely pubescent; young bracts $1.5(2) \mathrm{mm}$ long, densely pubescent, divided to base into linear lobes; developed bracts to 6-7 $\mathrm{mm}$ long, pubescent on upper and lower surface, with simple hairs; margin divided into 8-10 linear teeth $2 / 5-3 / 5$ of the bract length, subacute, finely ciliate and papillose, flowers solitary. Male flowers inconspicuous, hispidulous; pedicel to $1 \mathrm{~mm}$ long, buds c. $1 \mathrm{~mm}$ diameter, sometimes with sparse minute bright droplets. Female flowers sessile; sepals 3 , triangular, c. $1 \times 0.5 \mathrm{~mm}$, hispidulous-ciliate; ovary 1.5-2.4 mm diameter, densely pubescent; styles 


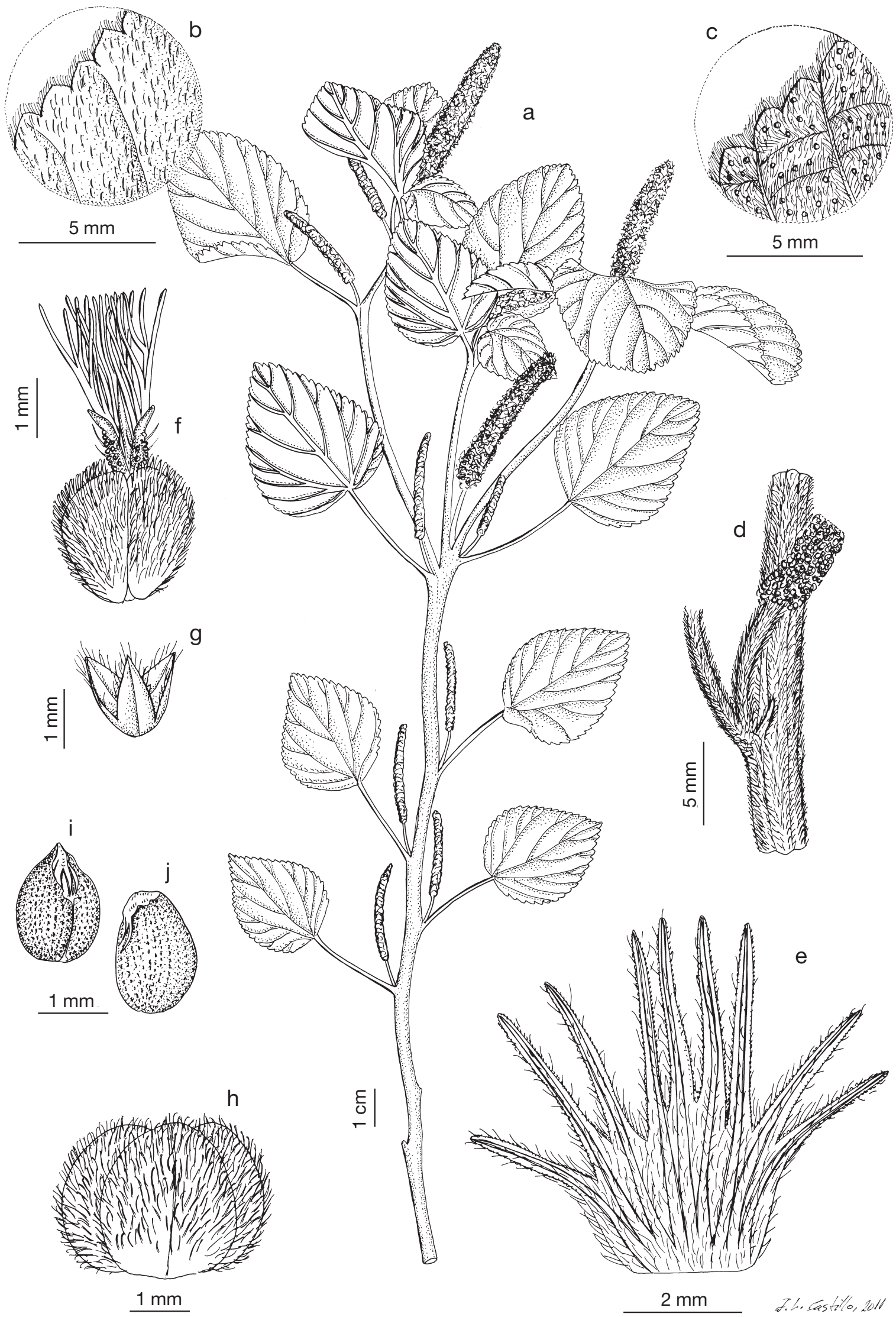

Fig. 5. Acalypha psamofila. a, flowering branch; $\mathbf{b}$, detail of the leaf upper surface and margin; $\mathbf{c}$, detail of the leaf lower surface; $\mathbf{d}$, detail of the petiole, male inflorescence and stipule; e, female developed bract; f, ovary and styles; g, calyx of female flower; $\mathbf{h}$, capsule; i, j, seeds. a-d, f, g, M. Nee 37935 (MA); e, h, i, j, M. Nee 48480 (MA). 
3.5-5 mm long, thickened at base, hispidulous, each branched into 6-7 slender segments. Capsule c. $3 \mathrm{~mm}$ diameter, densely pubescent; seeds piriform, $2 \times 1.4 \mathrm{~mm}$, minutely foveolate.

Etymology: The specific epithet refers to the characteristic habitat of this plant, which grows in sandy soils. Most of the herbarium specimens studied have abundant sand grains attached to the branches and leaves.

Distribution: Western South America, Bolivian endemism. BOLIVIA: Chuquisaca and Santa Cruz (10 collections examined). Chiquitanía region, $375-480 \mathrm{~m}$. Grassy stabilized or active sand dunes (Fig. 4f).

Acalypha psamofila can be included in a recently defined Acalypha sect. Communes (Pax \& K. Hoffm.) Cardiel, P. Muñoz \& Muñoz Garm., a complex group of species widespread in the northern part of the Southern Cone (Cardiel \& al., 2013). Within this group the new species is close to A. vellamea Baill. of Brazil and Paraguay. Acalypha psamofila can be distinguished from $A$. vellamea mainly by its leaves with petioles to $4.5 \mathrm{~cm}$ long (vs. to $0.5 \mathrm{~cm}$ long in $A$. vellamea) and its inconspicuous stipules, to $2.5 \mathrm{~mm}$ long (vs. to $13 \mathrm{~mm}$ long in A. vellamea). Among the Bolivian species it is related to $A$. communis subsp. communis and $A$. variabilis. Acalypha psamofila can be distinguished from $A$. communis mainly by its whitish indument on young branches and the lower leaf surface, and its female bracts without glandular hairs (vs. no whitish indument and female bracts with glandular hairs in A. communis). Acalypha psamofila differs from $A$. variabilis by lacking the conduplicate leaves with bright exudate characteristic of this species.

Additional specimens examined: BOLIVIA. Chuquisaca: Prov. Luis Calvo, El Salvador, Laguna Seca, campo Quemado, [203's, 632 21'W], 09 Dec1992, J. Penseiro \& G. Marino 125 (NY), 4397 (NY). Santa Cruz: Prov. Andrés Ibáñez, ca. $15 \mathrm{~km}$ al Norte de Santa Cruz, por el nuevo aeropuerto Viru

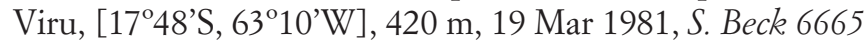
(DAV, LPB, MA); In Hecken um Sta. Cruz, 450 m, T. Herzog 1529 (S, W); W of Santa Cruz to Montero, $17^{\circ} 39^{\prime} \mathrm{S}, 63^{\circ} 10^{\prime} \mathrm{W}$, 375 m, 26 Feb 1988, M. Nee 36389 (MO, NY); along río Chore-Chore, $17^{\circ} 56^{\prime} \mathrm{S}, 6^{\circ} 06^{\prime} \mathrm{W}, 380 \mathrm{~m}$, 22 Jan 1989, M. Nee

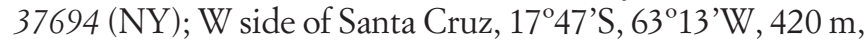
03 Dec 1989, M. Nee 37935 (K, MA, MO, NY); 7 km SE of Naranjillos, $18^{\circ} 02^{\prime} \mathrm{S}, 63^{\circ} 12^{\prime} \mathrm{W}, 480 \mathrm{~m}, 30$ Sep 1990, M. Nee 38990 (LPB, MA, MO, NY); along quebada Peji, 1757'S, $63^{\circ} 11^{\prime} \mathrm{W}, 440$ m, 11 Dec 1994, M. Nee 45836 (NY); El Pari

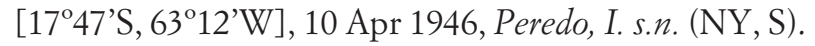

\section{Acalypha reflexa Müll. Arg., Linnaea 34: 33 (1865).}

TYPE: [Peru] In Peruvia, J.A. Pavón s.n. (holotype, GDC, [G00324473, negative F: 7144]!).

Acalypha mandonii Müll. Arg. Linnaea 34: 162 (1865). Ricinocarpus mandonii (Müll. Arg.) Kuntze, Revis. Gen. Pl. 2: 618 (1891).

TYPE: [Bolivia: La Paz] In Boliviae Andinae prov. Larecaja prope Sorata, 2600-2700 m, 1858-1859, G. Mandon 1071 (holotype, G-DC [G00324474, negative F 7141]!; isotypes, BM!, F[2 sheets]!, K!, MA!, NY[2 sheets]!), P[3 sheets]!, $\mathrm{S} !)$.
Acalypha soratensis Pax \& K. Hoffm. in Engl., Pflanzenr. IV. 147-16 (Heft 85): 126 (1924).

TYPE: [Bolivia: La Paz] Sorata, May 1892, M. Bang 1316 (lectotype, designated here, K [K000600509]!, isolectotypes, BM!, F!, K!, M!, MO!, NY[2 sheets]!, US). Other type collection: [Bolivia: La Paz] Sorata, 8000 ft, Feb 1886, H.H. Rusby 1257 ["ex parte" in protologue] (BM!, GH!, MO!, NY[4 sheets]!, US!).

Iconography: Fig. 6.

Distribution: Western South America (Peru and Bolivia). PERU: Cajamarca and Junín (7 collections examined). BOLIVIA: La Paz and Santa Cruz (12 collections examined). Andean region, 1900-3000 m. Dry forests, rocky slopes (Fig. 7a). References: Cardiel (2007).

In previous treatments, Acalypha mandonii has been considered the accepted name for this species, and $A$. reflexa its synonym. However, $A$. mandonii was described in the $2^{\text {nd }}$ fascicle of the $34^{\text {th }}$ volume of the Linnaea journal, while $A$. reflexa was described in the $1^{\text {st }}$ fascicle of the $34^{\text {th }}$ volume. According to Foster (1962), fascicle 1 was published in March 1865 and fascicle 2 in December 1865. Therefore the first name should have priority. The holotype of $A$. reflexa, from G-DC, shares the herbarium sheet with the type specimen of $A$. contermina Müll. Arg. (J.A. Pavón s.n.).

30. Acalypha salicifolia Müll. Arg., Flora 47: 438 (1864). Ricinocarpus salicifolius (Müll. Arg.) Kuntze, Revis. Gen. P1. 2: 618 (1891).

TYPE: [Ecuador] In Andibus Ecuadorensibus, R. Spruce 4963 (holotype, W; isotypes, F[fragment ex W]!, K[2 sheets]!).

Acalypha macbridei I.M. Johnst., Contr. Gray Herb. 75: 28 (1925).

TYPE: [Peru: Huánuco] Pampayacu, hacienda at mouth of Rio Chiao, J.F. Macbride 5093 (holotype, F!; isotypes, GH!, $\mathrm{K}$ !, US!).

Iconography: Fig. 8.

Distribution: Western South America (Ecuador and Peru). PERU: Amazonas, Huánuco, Junín, Loreto and Pasco (19 collections examined). Not found in Bolivia. Amazonian region and Andean foothills, (300)700-1600 m. Rain forests and montane forests (Fig. 7b).

References: Brako (1993), Cardiel (2007), Cardiel \& Muñoz (2012a), Muñoz-Rodríguez \& al. (2014).

31. Acalypha salicina Hutch. ex Cardiel, Nordic J. Bot. 22(5): 627 (2003).

TYPE: Peru: Puno, río Távara, border of river, $400 \mathrm{~m}, 13^{\circ}$ 21'S, 69' 40'W, 20 May 1992. A. Gentry, C. Reynel, R. Ortiz EP. Núñez 76917 (holotype, MO!; isotype, MA!).

Iconography: Cardiel, Nordic Journal of Botany 22(5): 628, fig. 1. 2003.

Distribution: Western South America, Peruvian endemism. PERU: Cuzco and Puno (5 collections examined). Amazonian region and Andean foothills, 150-1100 m. River banks (Fig. 7b).

References: Ulloa Ulloa \& al. (2004), Cardiel (2007). 


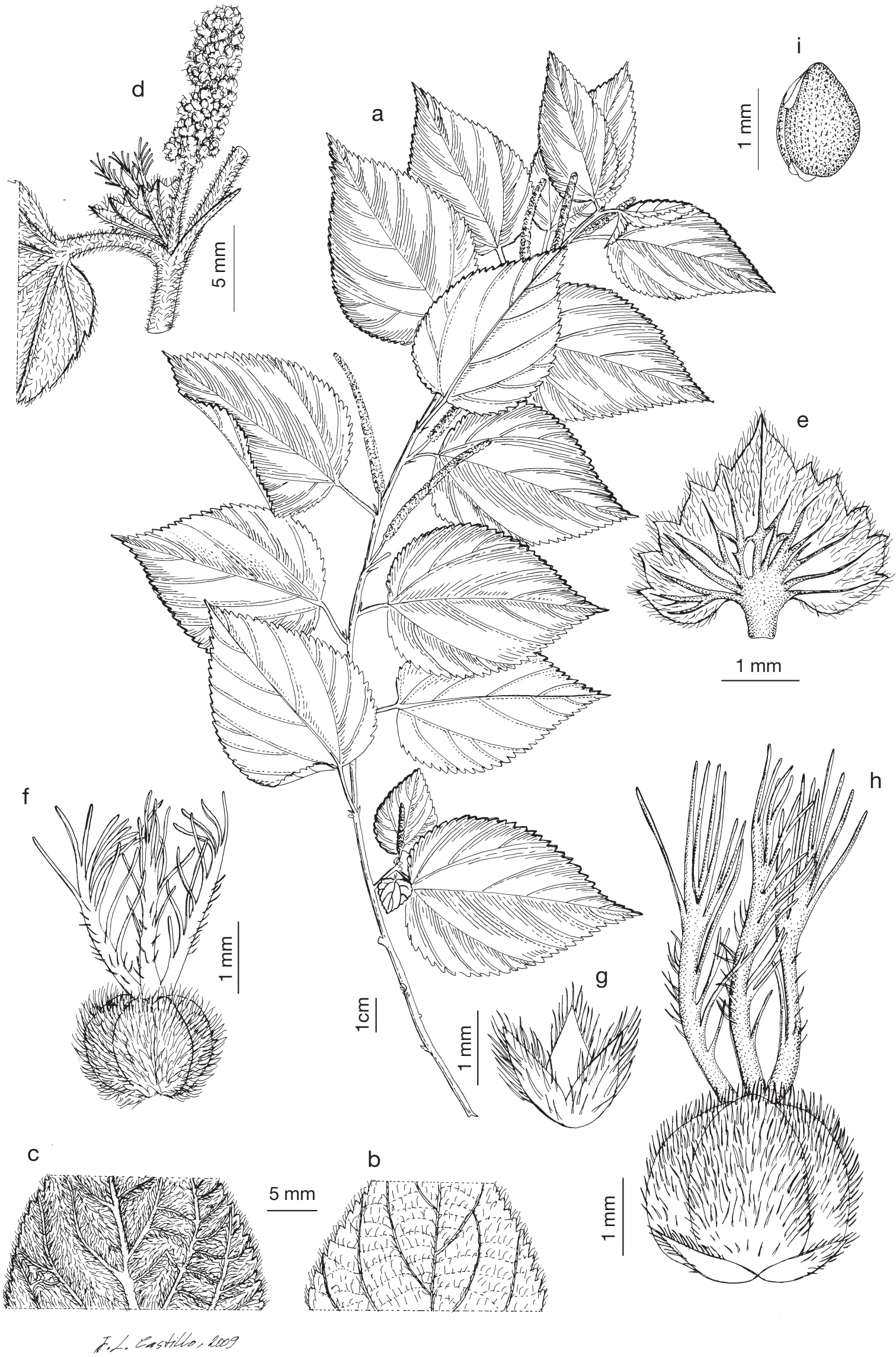

Fig. 6. Acalypha reflexa. a, flowering branch; $\mathbf{b}$, detail of the leaf upper surface; $\mathbf{c}$, detail of the leaf lower surface; $\mathbf{d}$, detail of the leaf base, androgynous inflorescence and stipule; e, female developed bract; f, ovary and styles; g, calyx of female flower; $\mathbf{h}$, capsule and styles; i, seed. a-i, J.R.L. Wood 8161 (LPB). 

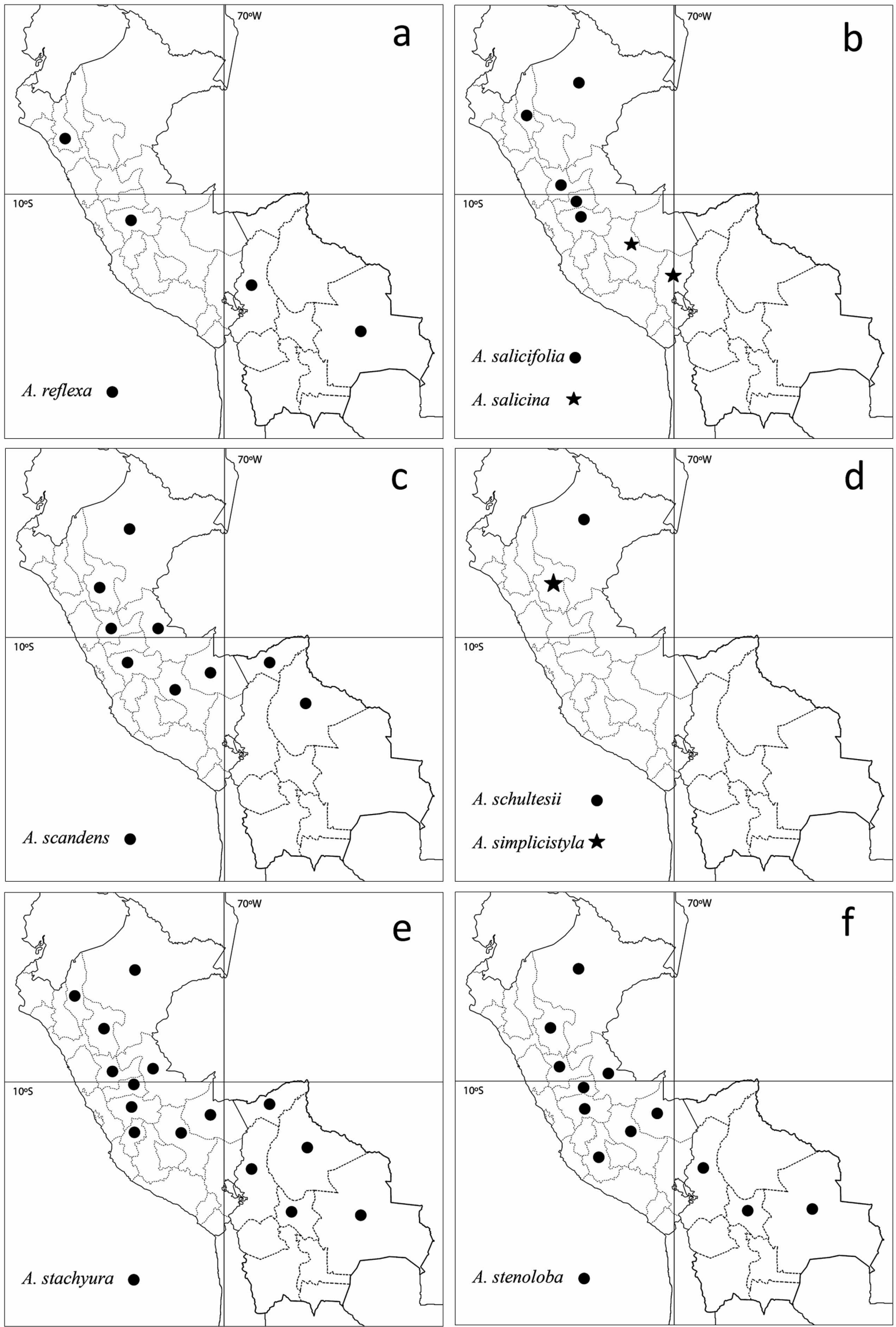

Fig. 7. Maps showing the Bolivian and Peruvian distributions of selected Acalypha species. a, A. reflexa; b, A. salicifolia and A. salicina; c, A. scandens; d, A. schultesii and A. simplicistyla; e, $A$. stachyura; $\mathbf{f}, A$. stenoloba. 
32. Acalypha scandens Benth., Hooker's J. Bot. Kew Gard. Misc. 6: 329 (1854). Ricinocarpus scandens (Benth.) Kuntze, Revis. Gen. Pl. 2: 617 (1891).

TYPE: [Brazil: Pará] On the island of the Amazon opposite Santarem, R. Spruce 1000 (holotype, K!). Other type collection: [Surinam] F.W.R. Hostmann 990 (W!).

Iconography: Cardiel, Flora de Colombia 15: 109, fig. 24. 1995.

Distribution: Northern South America, western South America and Brazil. PERU: Cuzco, Huánuco, Junín, Loreto, Madre de Dios, San Martín and Ucayali. (104 collections examined). BOLIVIA: Beni and Pando (7 collections examined). Amazonian region, 100-500(900) m. Seasonally inundated forests (Fig. 7c).

References: Brako (1993), Vásquez (1997), Cardiel (2007), Valenzuela \& al. (2007), Cardiel \& Muñoz (2012a).

33. Acalypha schultesii Cardiel, Anales Jard. Bot. Madrid 52(2): 155 (1995).

TYPE: Colombia: Amazonas. N'uma ilha à margen da lagoa Dolphim (Loreto-Yaco), 30 Oct 1946, R.E. Schultes E Black 46-250 (holotype, F!).

Iconograpby: Cardiel, Anales del Jardín Botánico de Madrid 52(2): 156, fig. 2. 1995.

Distribution: Western South America (Colombia and Peru). PERU: Loreto. Not found in Bolivia. We only know of two Peruvian collections, from the Amazonian region, at about 180 m. River banks (Fig. 7d).

References: Cardiel (2007).

34. Acalypha simplicistyla Cardiel, Nordic J. Bot. 22(5): 629 (2002).

TYPE: Peru: San Martín. Prov. Mariscal Cáceres, Distrito Tocache Nuevo, quebrada de Saule Chico, margen derecha del río Huallaga, 7 Sep 1970, J. Schunke 4347 (holotype, US!; isotypes, F!, GH!, MO!, USM).

Iconography: Cardiel, Nordic Journal of Botany 22(5): 630, fig. 2. 2003.

Distribution: Western South America, Peruvian endemism. PERU: San Martín (3 collections examined). Andean foothills, 400-1000 m. Rain forests (Fig. 7d).

References: Ulloa Ulloa \& al. (2004), León \& al. (2006), Cardiel (2007).

35. Acalypha stachyura Pax, Repert. Spec. Nov. Regni Veg. 7: 110 (1909).

TYPE: [Bolivia: La Paz] Charopampa und San Carlos bei Mapiri, 750 m, Aug-Nov 1909. O.Buchtien 1315 (lectotype, M!, designated by Cardiel \& Muñoz (2012a: 13); isolectotype, US!). Other type collections: O. Buchtien 1307 (WRSL, US!), 1314 (US!).

Acalypha macrophylla Ule, Verh. Bot. Vereins Prov. Brandenburg 50: 79 (1908). Nom. illeg., non Kunth 1917.

TYPE: [Peru: Loreto] feuchte Niederung bei Tarapoto, $E$. Ule 6656 (lectotype, designated here, G!; isolectotypes, B [presumed destroyed, negative F 5299!], K!).

Iconography: Cardiel, Flora de Colombia 15: 80, fig. 21. 1995.
Distribution: Western South America and Brazil. PERU: Amazonas, Cuzco, Huancavelica, Huánuco, Junín, Loreto, Madre de Dios, Pasco, San Martín and Ucayali. (78 collections examined). BOLIVIA: Beni, Cochabamba, La Paz, Pando and Santa Cruz (98 collections examined). Amazonian region and Andean foothills, 100-1000(1500) m. Seasonally inundated rain forests (Fig. 7e).

References: Brako (1993), Cardiel (2007), Vásquez (1997, 2010).

\section{Acalypha stenoloba Müll. Arg., Flora 55: 41 (1872).}

TYPE: [Peru] Habitat in Peruvia. T. Haenke s.n. (holotype, M!, negative F 19517).

Acalypha capillaris Rusby, Mem. Torrey Bot. Club 4(3): 257 (1895).

TYPE: [Bolivia: La Paz] Yungas, 1890, M. Bang 676 (lectotype, designated here, NY [246103]!; isolectotypes, BM!, F!, GH!, K!, MO[2 sheets], NY![3 sheets], US![3 sheets]). Other type collection: [Bolivia: Santa Cruz] Santa Cruz, Feb 1865. R. Pearce s.n. (K!).

Ricinocarpus gracilis var. arboreus Kuntze, Revis. Gen. Pl. 3(2): 291 (1898), syn. nov.

TYPE: [Bolivia: Santa Cruz] Rio Juntas, 1600 m, O. Kuntze s.n (lectotype, designated here, NY!). Other type collection: [Bolivia: Santa Cruz] Santa Cruz, 350 m, O. Kuntze s.n.

Acalypha brittoni Rusby, Bull. Torrey Bot. Club 28: 303 (1901).

TYPE: [Bolivia: La Paz] Mapiri, 5000 ft, May 1886, M. Bang 1260 (lectotype, designated here, NY [312936]!; isolectotypes, NY!, US!).

Acalypha grandispicata Britton ex Rusby, Bull. Torrey Bot. Club 28: 304 (1901).

TYPE: [Bolivia: La Paz] Prov. Larecaja, Mapiri, $5000 \mathrm{ft}$, May 1886. M. Bang 1261 (lectotype, designated here, NY [246113]!; isolectotype, NY!)

Acalypha lechleri Britton ex Rusby, Bull. Torrey Bot. Club 28: 304 (1901).

TYPE: [Bolivia: La Paz] Prov. Nor Yungas, Unduavi, 8000 ft, Oct. 1885. M. Bang 2610 (lectotype, designated here, US [931764]!; isolectotypes, NY[2 sheets]!). Other type collections: [Bolivia: La Paz] H.H. Rusby 1420 (NY!); [Peru] Lechler 2408 (K!).

Acalypha lucida Rusby, Bull. New York Bot. Gard. 4: 444 (1907).

TYPE: [Bolivia] M. Bang 2560 (lectotype, designated here, NY [246122]!; isolectotypes, BM!, F!, G!, K!, M!, MICH!, MO, NY! [3 sheets], US!). Other type collection: [Bolivia] M. Bang 2561 (BM!, F!, MICH!, MO, NY![2 sheets], M!, US!).

Acalypha baenitzii Pax, Repert. Spec. Nov. Regni Veg. 5: 227 (1908).

TYPE: [Bolivia: La Paz] Surupaya [Sirupoya] bei Yanacachi, 2100 m, 19 Jun 1906. O. Buchtien 376 (holotype, US [1158271]!). 


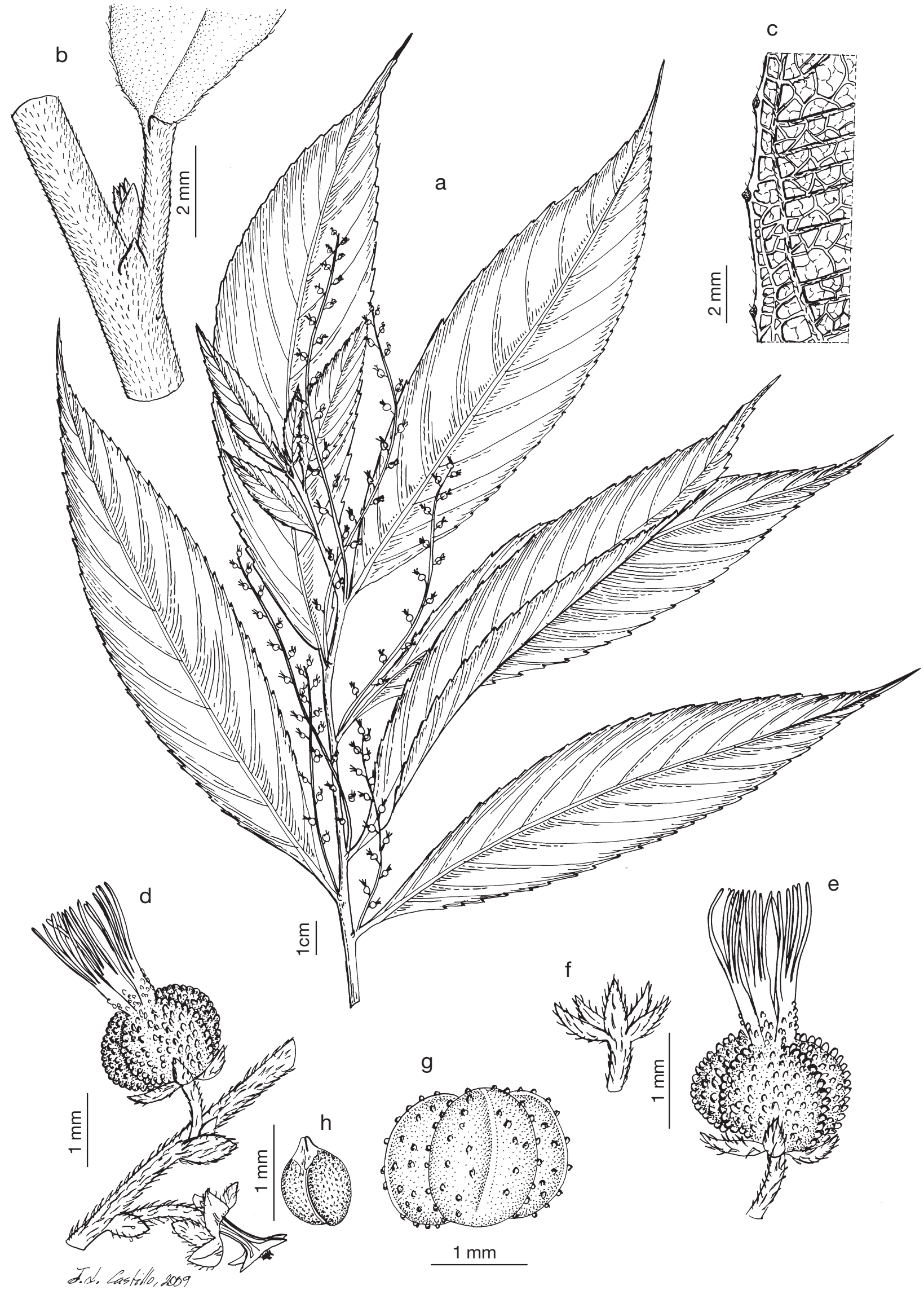

Fig. 8. Acalypha salicifolia. a, flowering branch; $\mathbf{b}$, detail of the leaf base and stipule; $\mathbf{c}$, detail of the margin and leaf lower surface; $\mathbf{d}$, detail of female inflorescence; e, female flower; f, calyx of female flower; $\mathbf{g}$, capsule; h, seed. a-h, J. Jaramillo \& al. 13069 (SEL). 
Acalypha ovata Pax \& K. Hoffm., Meded. Rijks-Herb. 40: 23 (1921).

TYPE: [Bolivia: Cochabamba] Locotal, 1600-1900 m, T. Herzog 2255 (holotype, Z [15840]!

Acalypha douilleana Rusby, Mem. New York Bot. Gard. 7: 285 (1927).

TYPE: [Bolivia: La Paz] Prov. Inquisivi, Cañamina, 4000 ft, 15 Jul 1921, H.H. Rusby 74 (holotype, NY[00246104]!; isotypes, $\mathrm{K}$ !, MICH!, US!).

Acalypha engenifolia Rusby, Mem. New York Bot. Gard. 4: 443 (1907).

TYPE: [Bolivia: La Paz] Near Coroico, 03 Aug 1894. M. Bang 2368 (lectotype, designated here, NY [246107]!; isolectotypes, C!, F!, G!, K!, M!, MICH!, MO!, NY [3 sheets]!, US!, Z!).

Iconography: Fig. 9.

Distribution: Western South America (Bolivia and Peru). PERU: Ayacucho, Cuzco, Huánuco, Junín, Loreto, Madre de Dios, Pasco, San Martín and Ucayali (57 collections examined). BOLIVIA: Cochabamba, La Paz and Santa Cruz (98 collections examined). Andean foothills and Amazonian region, (250)500-2100(2500) m. Rain forests and montane forests (Fig. 10f).

References: Vargas (1993), Cardiel (2007), Vásquez (2010).

Although poorly preserved, Ricinocarpus gracilis var. arboreus appears to match $A$. stenoloba and therefore we propose treating the former name as a synonmy of $A$. stenoloba.

37. Acalypha stricta Poepp. in Poepp. \& Endl., Nov. Gen. Sp. Pl. 3:21, pl. 225 (1845). Ricinocarpus strictus (Poepp.) Kuntze, Revis. Gen. Pl. 2: 618 (1891).

TYPE: [Peru: Lima] Crescit in fruticetis montanis Peruvuae orientalis ad Pampayaco [Pampayacu], Jul, E. Poepppig s.n. (holotype, W!; isotype, F!).

Acalypha benensis Britton ex Rusby, Bull. Torrey Bot. Club 28: 304 (1901).

TYPE: [Bolivia: Beni] Prov. Vaca Díez, junction of Rivers Beni and Madre de Dios, Aug 1886, H.H. Rusby 1264 (lectotype, designated here, NY [246097]!; isolectotypes, BM!, GH!, K!, MA!, MO!, NY[2 sheets]!, P!, US[2 sheets]! W!).

Acalypha mapirensis Pax, Repert. Spec. Nov. Regni. Veg. 7: 110 (1909).

TYPE: [Bolivia: La Paz] Charopampa bei Maripi, $570 \mathrm{~m}$. O. Buchtien 1308 (lectotype, designated here, US [1158887]!). Other type collections: [Bolivia: La Paz] loc. cit., O. Buchtien 1310 (B[presumed destroyed, negative F 5301!], F!, MO[2 sheets]!, 1311 (US[3 sheets]!).

Acalypha mapirensis var. pubescens Pax \& K. Hoffm., in Engl., Pflanzenr. 147, 16(Heft 85): 65 (1924).

TYPE: [Brazil] Alto Amazonas: Rio Acre, Seringal São Francisco, E. Ule 9535 (lectotype, designated here, NY [246129]!; isolectotypes, B [presumed destroyed]).

Acalypha variegata Rusby, Mem. New York Bot. Gard. 7: 285 (1927).
TYPE: [Bolivia: La Paz] Prov. Inquisivi, Cañamina, 4000 $\mathrm{ft} 30$ Jul 1921. E.O. White s.n. (holotype, NY[312942]!).

Acalypha bopiana Rusby, Mem. New York Bot. Gard. 7: 287 (1927).

TYPE: [Bolivia: La Paz] cataracts of the Bopi River, 3000 ft, 3 Sep 1921. H.H. Rusby 478 (holotype, NY[312935]!; isotypes, K!, MA!, MICH!).

Acalypha tomentosula Ule, Verh. Bot. Vereins Prov. Brandenburg 50: 79 (1908).

TYPE: [Peru: Loreto] bei Tarapoto, Dec 1902, E. Ule 6637 (holotype, K [K000600518]!).

Iconography: Poeppig \& Endlicher, Nova genera ac species plantarum 3, pl. 225. 1845.

Distribution: Brazil, Western South America (Peru and Bolivia). PERU: Amazonas, Ayacucho, Cuzco, Huánuco, Junín, Lima, Loreto, Madre de Dios, Pasco, Piura, Puno, San Martín and Ucayali. (222 collections examined). BOLIVIA: Beni, Cochabamba, La Paz, Pando and Santa Cruz (167 collections examined). Coastal, Amazonian, Andean and Chiquitanía regions, (100)300-2000(2500) m. Rain forests, shrublands and disturbed areas (Fig. 10a).

References: Brako (1993), Killeen \& al (1993), Jardim \& al. (2003), Cardiel (2007), Valenzuela \& al. (2007), Vásquez (2010), Berry \& al. (2013).

Acalypha stricta is the more frequently collected species in Peru and Bolivia.

38. Acalypha subcastrata F. Aresch., Pl. Itin. Eugeniae: 137 (1910).

TYPE: [Ecuador] On Puna i Guayaquil viken, N.J. Andersson 160 (lectotype, S[S-R-7773]!, designated by Cardiel \& Muñoz (2012: 14); isolectotype, S[08-1622]!).

Iconography: Fig. 11.

Distribution: Western South America (Ecuador and Peru). PERU: Cajamarca, Lambayeque, Piura and Tumbes $(9$ collections examined). Not found in Bolivia. Coastal region, 150400(1200) m. Dry forests and disturbed areas (Fig. 10b).

References: Cardiel (2007), Cardiel \& Muñoz (2012a).

39. Acalypha variabilis Klotzsch ex Baill., Adansonia, 5: 226 (1865). A. variabilis var. typica Baill., Adansonia 5: 227 (1865).

TYPE: [Brazil] Brasilia, F. Sellow s.n. (lectotype, P [635221]!, designated by Cardiel \& al. (2013: 1301); isolectotypes, P[635222]!, B [presumed destroyed].

Iconography: Unknown.

Distribution: Western South America (Bolivia), Brazil and southern South America (Argentina, Paraguay and Uruguay). BOLIVIA: Beni and Santa Cruz (23 collections examined). Not found in Peru. Chiquitanía region, 250-500(1000) m. Grasslands, shrublands, rocky places and disturbed areas (Fig. 1b).

References: Cardiel \& al. (2013).

Acalypha variabilis has been usually treated as a synonym of A. communis. According to the recent revision of Acalypha sect. Communes (Cardiel \& al., 2013), A. variabilis should be considered a distinct species. 


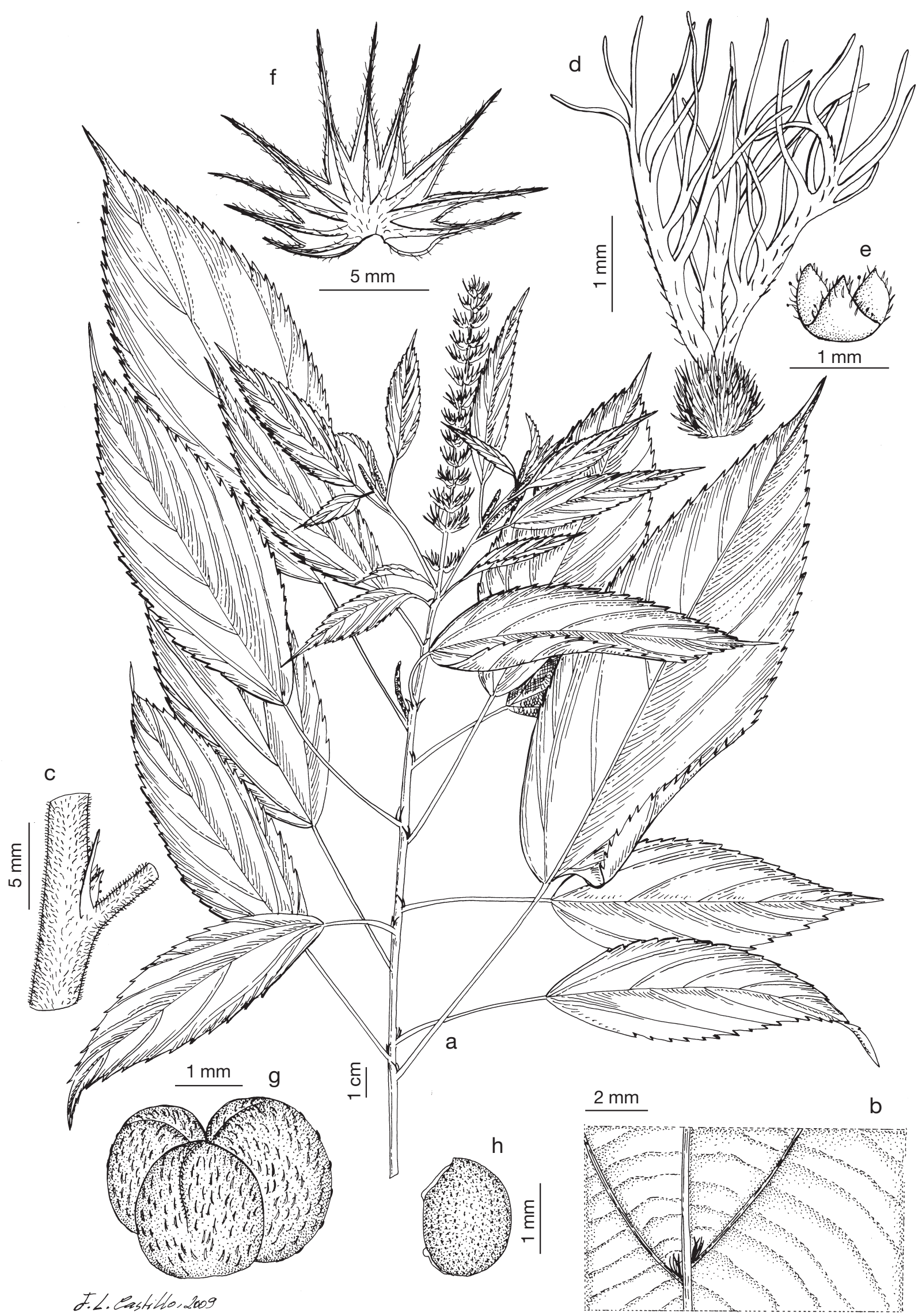

Fig. 9. Acalypha stenoloba. a, flowering branch; $\mathbf{b}$, detail of the leaf lower surface; $\mathbf{c}$, detail of the petiole and stipule; $\mathbf{d}$, ovary and styles; e, calyx of female flower; f, female developed bract; g, capsule; $\mathbf{h}$, seed. a-e, g, h, I. Vargas 2102 (MA), f, BMA 13 (MA). 

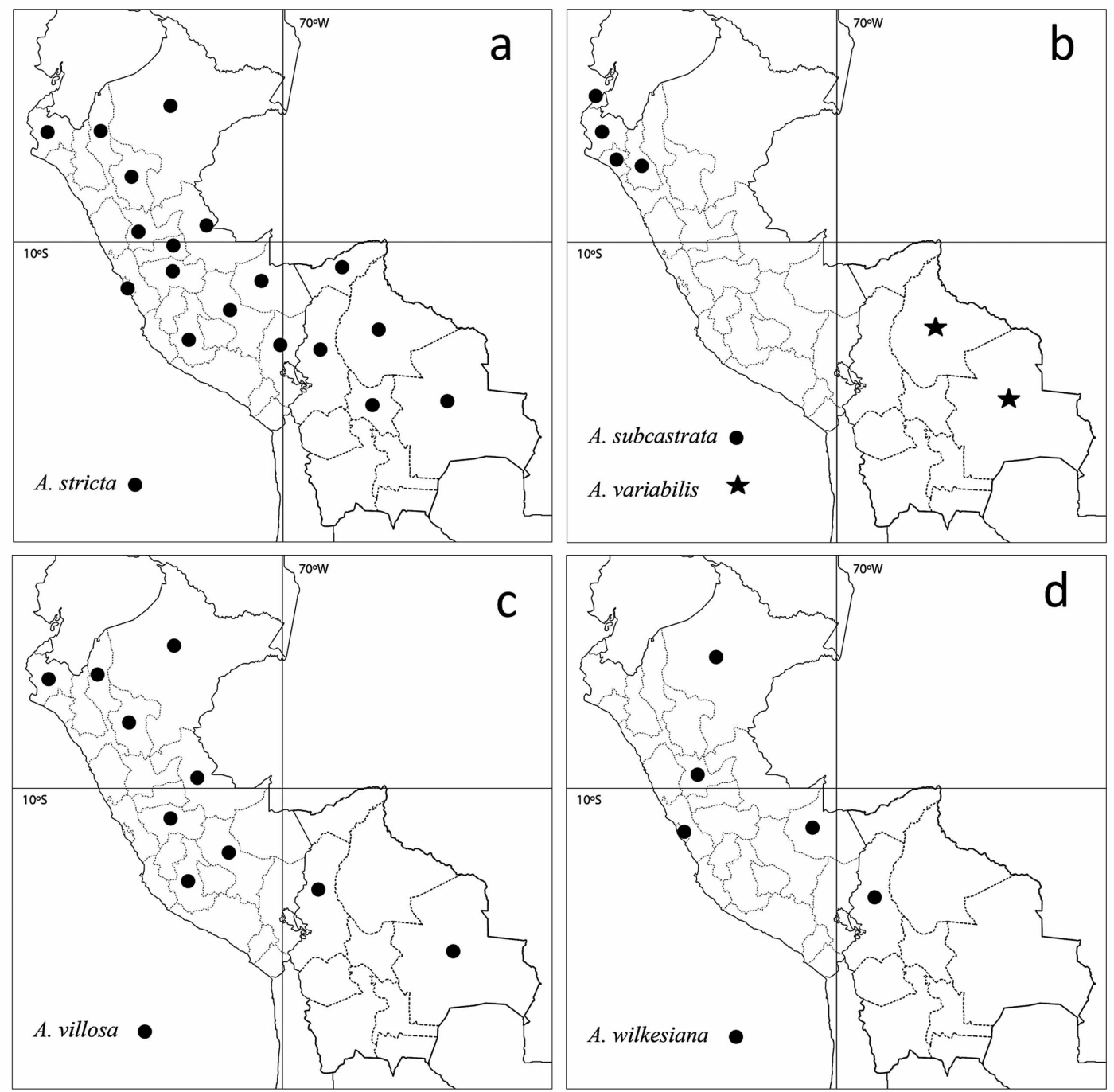

Fig. 10. Maps showing the Bolivian and Peruvian distributions of selected Acalypha species. a, A. stricta; $\mathbf{b}, A$. subcastrata and $A$. variabilis; c, $A$. villosa; d, A. wilkesiana.

40. Acalypha villosa Jacq., Enum. Syst. Pl. 32 (1760). Ricinocarpus villosus (Jacq.) Kuntze, Rev. Gen. Pl. 2: 618 (1891). Gymnalypha jacquinii (Jacq.) Griseb., Bonplandia (Hannover) 6: 2 (1858).

TYPE: [Colombia: Bolívar] Habitat Carthagenae in silvis \& sepibus. Lectotype, Tab. 183 fig. 16. in Jacq., Select. Stirp. Amer. Hist. (1763), designated by R.A. Howard (1989). Epitype, Tab. 47 in Jacq., Hort. Bon. Vindov. 3 (1776), designated by J.M. Cardiel (1995[1996]).

Acalypha villosa Jacq. var. tomentosa Müll. Arg., Linnaea 34: 8 (1865).

TYPE: [Peru] In Peruvia, J.A. Pavon s.n. (lectotype, GDC[G00324082] designated by Muñoz-Rodríguez \& al. (2014: 213); isolectotype, W!).

Acalypha villosa Jacq. var. latiuscula Pax \& K. Hoffm. in Engl., Pflanzenr. 147, 16 (heft. 85): 17 (1924).

TYPE: [Ecuador] Manabi, bei Hacienda El Recreo, H. Eggers 15616 (lectotype, K!, designated by Cardiel \& Muñoz (2012a); isolectotype, L!).
Acalypha williamsii Rusby Descr. S. Amer. Pl. 47 (1920). Nom. illeg. non Rusby (1912). Acalypha rusbyi Dorr, Brittonia 43(4): 226 (1991).

TYPE: [Bolivia: La Paz] Prov. Iturralde, San Buena Ventura, 20 Nov 1901, R.S. Williams 655 (holotype, NY[00246143]!; isotypes, NY!, K!).

Iconography: Jacquin, Hortus botanicus vindobinensis 3 , Tab. 47. 1776.

Distribution: Mexico, Central America, Caribbean, northern South America, western South America, Brazil, southern South America (Argentina, Paraguay). PERU: Amazonas, Ayacucho, Cajamarca, Cuzco, Junín, Loreto, Piura, San Martín and Ucayali (31 collections examined). BOLIVIA: La Paz and Santa Cruz (46 collections examined). Amazonian, Andean and Chiquitanía regions, 260-1500(2200) m. Rain forests, gallery forests and river banks (Fig. 10 c).

References: Brako (1993), Berry (2007), Cardiel (2007), Cardiel \& Muñoz (2012a).

The name Acalypha williamsii had been already used eight years before by Rusby to describe a different species $(A$. williamsii Rusby [1912] = A. macrostachya Jacq.). 

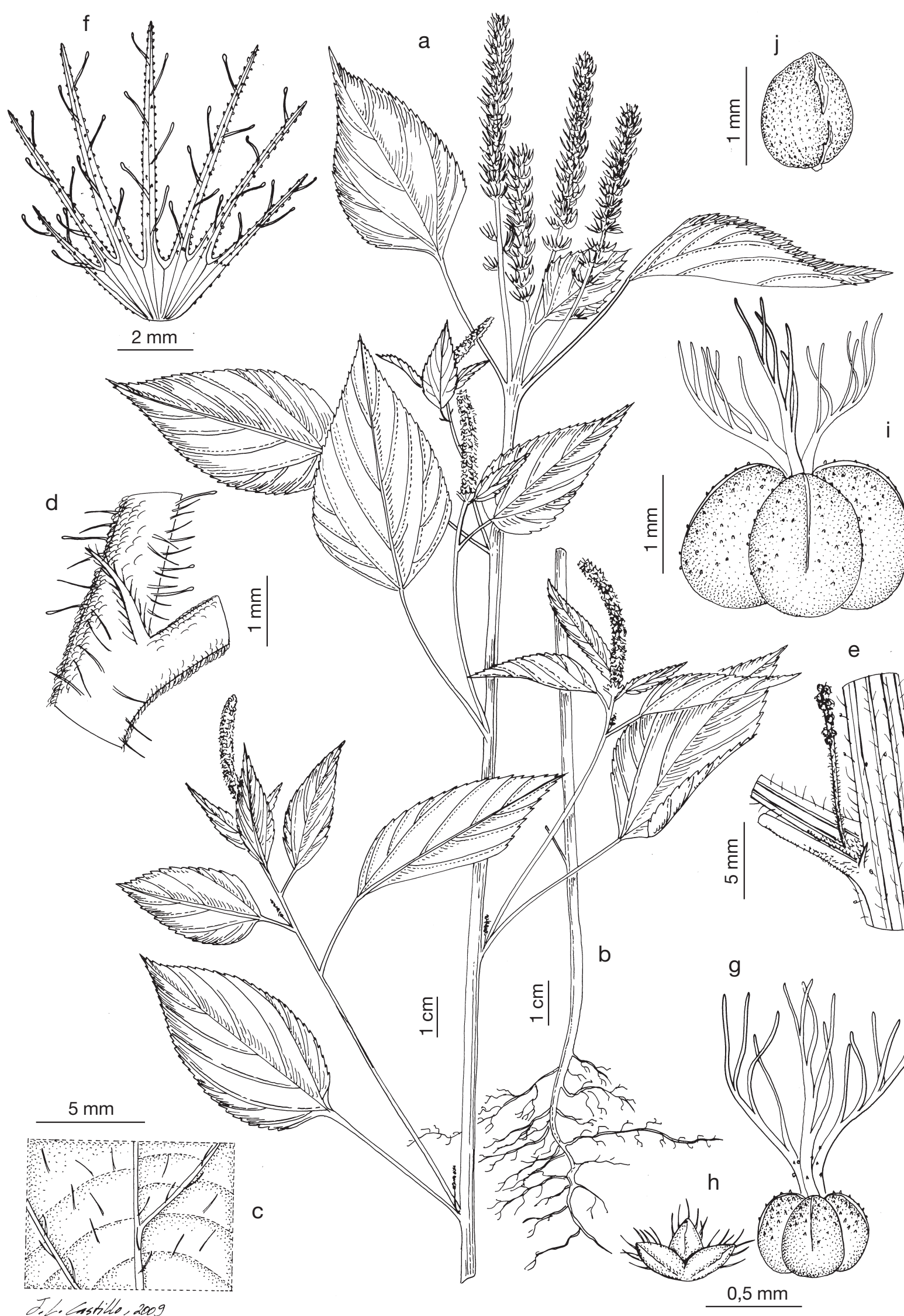
41. Acalypha wilkesiana Müll. Arg. in DC., Prodr. 15(2): 817 (1866). Ricinocarpus wilkesianus (Müll. Arg.) Kuntze, Revis. Gen. Pl. 2: 618 (1891). Acalypha amentacea Roxb. subsp. wilkesiana (Müll. Arg.) Fosberg, Smithsonian Contr. Bot. 45: 10 (1980).

TYPE: [Fiji] In insulis Fidji (U.S. Expl. Exped. Under. Capt. Wilkes), B.C. Seeman 22 (holotype, G-DC!; isotypes, $\mathrm{GH}$ !, K[2 sheets]!, US[2 sheets]!).

Iconography: Pérez-Arbeláez, Flora medicinal de Colombia, fig. 259. 1978.

Distribution: Native to the Polynesian island of Fiji, Acalypha wilkesiana is used as an ornamental in gardens throughout the tropics and frequently appears to be naturalized. PERU: Huánuco, Lima, Loreto and Madre de Dios (10 collections examined). BOLIVIA: La Paz (one collection examined). Coastal, Amazonian and Andean regions, 200-2000 m. Disturbed areas (Fig. 10d).

References: Cardiel (2007), Valenzuela \& al. (2007), Cardiel \& Muñoz (2012a).

\section{DOUBTFUL AND EXCLUDED NAMES}

Acalypha brachyclada Müll. Arg. in DC, Prodr. 15(2): 862 (1866).

TYPE: "In Peruvia aut in Mexico", J.A. Pavón s.n. (G-DC, foto $\mathrm{F} 8505$ !).

We only know the type collection, which probably comes from Mexico, as indicated in the original publication.

Acalypha contermina Müll. Arg., Linnaea 34: 46 (1865).

TYPE: "In Peruvia", J.A. Pavón. s.n. (G-DC[G00324474], negative F 7141!).

We only know the type collection, which probably does not come from Peru but from Mexico. This plant seems very close to some Mexican species still little known. This still requires a detailed study. Müller Argoviensis (1865) indicated that it is close to $A$. schiedeana Schltdl. The herbarium sheet from G-DC is mixed with the type specimen of $A$. mandonii Müll. Arg. (G. Mandon 1071).

\section{Acalypha cuprea Herzog, Repert. Spec. Nov. Regni Veg. 7:} 60 (1909).

TYPE: [Bolivia: Santa Cruz] Prov. Velasco "Im Gebüsch der Quebrada de San Lorenzo" 400 m, Jul 1907, T. Herzog 429 (?).

We could not find the type collection in the reviewed herbaria. Most of the Theodor Herzog's collections were deposited in the B herbarium. The cited specimen was probably destroyed in the herbarium fire of 1945 . According with the original description this taxon is closer to A. communis Müll. Arg. subsp. communis and probably should be treated as synonym of this taxon.

Acalypha jubifera Rusby, Descr. S. Amer. Pl. 48 (1920).

TYPE: [Bolivia] no locality or date, M. Bang s.n. (NY?).

We could not find the type collection in the reviewed herbaria. Rusby described this species based on a specimen without female flowers, so the original description does not provide enough information to determine the identity of this taxon.

\section{ACKNOWLEDGEMENTS}

This work was partly financed by the Spanish Government through the research project EUI 2008-0388, and by the Universidad Autónoma de Madrid (Spain) and the Regional Government (Comunidad de Madrid), through the research project CCG07-UAM/AMB-1453. This research also received support from the SYNTHESYS Project http://www.synthesys.info /which is financed by European Community Research Infrastructure Action under the FP7 "Capacities" Program. We kindly thank the curators and staff of the aforementioned herbaria for facilitating the study of their specimens, especially to Caroline Loup, Bruno Wallnöfer, Gill Challen and Maria Peña Chocarro of the P, W, K and BM herbaria, respectively for their kidness and efficiency. Finally, our appreciation to the reviewers of the manuscript for providing many usefull comments, and to Juan Castillo for his excellent drawings.

\section{REFERENCES}

Alverson, W.B.S., Moskovits, D.K. \& Shopland, J.S. 2000. Bolivia: Pando, Río Tahuamanu. Rapid Biological Inventories 1: 179.

Ambruster, W.S., Berry, P.E., Esser, H.-J., Gillespie, L.J., Hayden, W.J., Heald, S.V., Levin, G.A., Punt, W., Secco, R. de S. \& Webster, G.L. 2007. Euphorbiaceae. In: Funk, V.A., Berry, P.E., Alexander, S., Hollowell, T.H. \& Kelloff, C.L. Checklist of the Plants of the Guiana Shield (Venezuela: Amazonas, Bolivar, Delta Amacuro, Guyana, Surinam, French Guiana. Contributions from the United States National Herbarium 55:290300.

Arroyo, L. \& Villarroel, D. 2009. Las Plantas con Flores. 81-139. In: Arroyo, L. \& Churchill, S.P., Inventarios Bot. Área Bella Vista. Museo de Historia Natural Noel Kempff Mercado \& Missouri Botanical Garden, Santa Cruz de la Sierra.

Bacigalupo, N.M. \& Mulgura, M.E. 1999. Acalypha. In: Zuloaga, O. \& Morrone, O. (eds.), Catálogo de las plantas vasculares de la República Argentina 2: 590-592. Missouri Botanical Garden Press.

Balick, M.J., Nee, M.H. \& Atha, D.E. 2000. Checklist of the Vascular Plant of Belize with Common Names and Uses. Memoirs of the New York. Botanical Garden 85: 1-256.

Berry, P.E. 2007. Acalypha. In: Zuloaga, F.O., Morrone, O. \& Belgrano, M.J. (eds.), Catálogo de las Plantas Vasculares del Cono Sur (Argentina, Sur de Brasil, Chile, Paraguay y Uruguay) Pteridophyta, Gymnospermae y Monocotyledonae 1: 2010-2014. Missouri Botanical Garden Press.

Brako, L. 1993. Acalypha. In: Brako, L., Zarucchi, J.L. (eds.), Catálogo de las Angiospermas y Gimnospermas del Perú. Monographs in Systematic Botany from the Missouri Botanical Garden, St. Louis 45: 428-429.

Brako, L. \& Zarucchi, J.L. 1993. Catálogo de las Angiospermas y Gimnospermas del Perú. Monographs in Systematic Botany from the Missouri Botanical Garden, St. Louis 45.

Brummit, R.K. 2001. World geographical scheme for recording plant distributions, Edition 2. Plant Taxonomic Database Standards, No. 2. International Working Group on Taxonomic Databases for Plant Sciences (TDWG). Hunt Institute for Botanical Documentation Carnegie Mellon University, Pittsburgh.

Burger, W \& Huft, J.M. 1995. Family 113. Euphorbiaceae. In: Burger, W. (ed.), Flora Costaricensis. Fieldiana Botany 36: 1-169.

Cardiel, J.M. 1995. Acalypha (Euphorbiaceae). Flora de Colombia. Monografía n ${ }^{\circ} 15$. Universidad Nacional de Colombia y Real Jardín BotánicoCSIC, Madrid.

Cardiel, J.M. 1995[1996]. Tipificación de las especies de Acalypha (Euphorbiaceae) descritas por Jacquin. Anales del Jardín Botánico de Madrid 54: 230-233.

Cardiel, J.M. 1999. Contribuciones a la Flora de Venezuela: Revisión del género Acalypha (Euphorbiaceae). Acta Botanica Venezuelica 22(2): 255 324.

Cardiel, J.M. 2003. Two new species of Acalypha L. (Euphorbiaceae) from Peru. Nordic Journal of Botany 22(5): 627-631.

Cardiel, J.M. 2006. Two new species of Acalypha (Euphorbiaceae) from Peru and Bolivia. Nordic Journal of Botany 24: 167-172.

Cardiel, J.M. 2007. Catálogo de las especies peruanas de Acalypha Linnaeus (Euphorbiaceae). Fontqueria 55(50): 405-414. 
Cardiel, J.M. 2010. Acalypha. In: Campostrini, R. \& al. (eds.), Catálogo de plantas e fungos do Brasil 2: 963-964. Rio de Janeiro; Andrea Jakobsson Estúdio: Instituto de Pesquisas Jardim Botânico do Rio de Janeiro.

Cardiel, J.M. \& Muñoz-Rodríguez, P. 2012a. Synopsis of Acalypha (Euphorbiaceae) from Continental Ecuador. PhytoKeys 17: 1-17.

Cardiel, J.M. \& Muñoz, P. 2012b. Two new species of Acalypha (Euphorbiaceae) from Bolivia. Brittonia 64(4): 363-367.

Cardiel, J.M., Muñoz, P. \& Muñoz, F., 2013. Revised taxonomy and nomenclature of Acalypha sect. Communes (Euphorbiaceae), a complex group of species widespread in the north of Southern Cone. Taxon 62(6): 1296-1304

Cardiel J.M., Muñoz, P., Dorda, E. \& Pardo de Santallana, M. 2013. Acalypha Taxonomic Information System. http://www.acalypha.es (accessed 09 Dec. 2013).

Correa, M.D., Galdames, C. \& de Stapf, M.S. 2004. Catálogo de las Plantas Vasculares de Panamá. Universidad de Panamá \& Instituto Smithsonian de Investigaciones Tropicales.

Foster, R.C. 1958. A catalogue of the ferns and flowering plants of Bolivia. Contributions from the Gray Herbarium 184: 1-223.

Foster, R.C. 1962. Dates of publication of the journal Linnaea. Journal of the Arnold Arboretum 43: 400-409.

Gillespie, L.J. 1993. Euphorbiaceae of the Guianas: Annotated species checklist and key to the genera. Brittonia 45(1): 56-94.

Gillespie, L.J. 1997. Euphorbiaceae. In: Boggan, J, Funk, V., Kelloff, C., Hoff, M., Cremers, G. \& Feuillet, C. Checklist of plants of the Guianas (Guyana, Surinam, Frech Guiana). 2nd edition, 100-103. Centre for the Study of Biological Diversity. University of Guyana. Georgetown.

González, J. 2010. Acalypha. In: Hammel, B.E., Grayum, M.H., Herrera, C. \& Zamora, N., (eds.), Manual de Plantas de Costa Rica. Vol. V, Dicotiledóneas (Clusiaceae-Gunneraceae). Monographs in Systematic Botany from the Missouri Botanical Garden: 296-305.

Guantay, M.E., Slanis, A.C. \& Bulacio, E. 2008. Caracterización del indumento caulinar de Acalypha poiretti (Euphorbiaceae, Acalypheae). Lilloa 45(1-2): 61-65.

Hayden, W.J. \& Hayden, S.M. 2000. Wood anatomy of Acalyphoideae (Euphorbiaceae). International Association of Wood Anatomists Journal 21: 213-235.

Jardim, A., Killeen, T.J. \& Fuentes, A. 2003. Guía de los Árboles y Arbustos del Bosque Seco Chiquitano i-x, 1-324. Fundación Amigos de la Naturaleza Noel Kempff, Santa Cruz de la Sierra.

Killeen, T.J., García Estigarribia, E. \& Beck, S.G. (eds.). 1993. Guía de Árboles de Bolivia 1-958. Herbario Nacional de Bolivia \& Missouri Botanical Garden, La Paz.

Killeen, T.J. \& Schulenberg, T.S. 1998. A biological assessment of Parque Nacional Noel Kempff Mercado, Bolivia. RAP Working Papers 10: 1-372.

León, B., Riina, R. \& Berry, P. 2006. Euphorbiaceae endémicas del Perú. Revista Peruana de Biología 13(2): 295-301.

Levin, G.A. 1999. Acalypha. In: Berry. P.E., Yatskievych, K. \& Holst, B.K. (eds.), Flora of the Venezuelan Guayana 5: 81-85. Missouri Botanical Garden Press, St. Louis.

Levin, G.A. 2001b. Acalypha. In: Stevens, W.D., Ulloa, C., Pool, A. \& Montiel, O.M. (eds.), Flora de Nicaragua. Introducción, Gimnospermas y Angiospermas (Acanthaceae-Euphorbiaceae) 85(1): 842-852. Missouri Botanical Garden Press, St. Louis.

Levin, G.A. 2008. Acalypha. In: Hokche, O., Berry, P.E. \& Huber, O. (eds.), Nuevo catálogo de la Flora Vascular de Venezuela. Fundación Instituto Botánico de Venezuela Dr. Tobías Lasser, Caracas.

Macbride, J.F. 1951. Flora of Peru. Euphorbiaceae. Publications of the Field Museum of Natural History. Botanical series 13 part. IIIA(1): 1-288.

McNeill, J., Barrie, F.R., Buck, W.R., Demoulin, V., Greuter, W., Hawksworth, D.L., Herendeen, P.S., Knapp, S., Marhold, K., Prado, J., Prud'Homme van Reine, W.F., Smith, G.F., Wiersema, J.H. \& Turland, N.J. (eds. \& comps.). 2012. International Code of Nomenclature for algae, fungi and plants (Melbourne Code). Regnum Vegetabile 154.

Moraes, R.M. 1990. Lista preliminar de especies botánicas coleccionadas durante la Expedición Río Madre de Dios (Norte de Bolivia). Museo Nacional de Historia Natural (Bolivia) 10: 32-52.

Muñoz-Rodríguez, P., Cardiel, J.M., \& Atha, D. 2014. Acalypha subgenus Linostachys (Euphorbiaceae, Acalyphoideae): a global review. Phytotaxa 166(3): 199-221.

Murillo, J. 2004. Las Euphorbiaceae de Colombia. Biota Colombiana 5(2): 183-200.
Müller Argoviensis, J. 1866. Acalypha. In: De Candolle, A.P. Prodromus Systematis Naturalis Regni Vegetabilis 15(2): 799889.

Nelson, C.H. 2008. Catálogo de las plantas vasculares de Honduras. Espermatófitas. Departamento de Biología. Universidad Nacional Autónoma de Honduras.

Parker III, T.A. \& Bailey, B. 1991. A biological assessment of the Alto Madidi region and adjacent areas of Northwest Bolivia. RAP Working Papers 1: 1-108.

Pax, F.A. \& Hoffmann, K. 1924. Euphorbiaceae-Crotonoideae-AcalypheaeAcalyphinae. In: Engler, A. (ed.), Das Pflazenreich IV: 147 XVI (Heft. 85). Wilheln Engelman, Leipzig.

Pennington, T., Reynel, C. \& Daza, A. 2004. Illustrated guide to the Trees of Peru. DH Books. England.

Renvoize, S.A. 1998. Gramineas de Bolivia. The Royal Botanical Gardens, Kew.

Saravia, E.F. 1996. Estudio de la vegetación de las partes altas de las provincias Campero y Mizque (Cochabamba). Tesis. Universidad Mayor de San Simón, Cochabamba.

Serrano, M. \& Terán, J. 1998 [2000]. Identificación de especies vegetales en Chuquisaca. 1-129. PLAFOR, Intercooperación, Fundación Ceibo, Sucre.

Steinmann, V.W. 2002. Diversidad y endemismo de la familia Euphorbiaceae en México. Acta Botánica Mexicana 61: 61-93.

Steinmann, V.W. \& Levin G.A. 2011. Acalypha herzogiana (Euphorbiaceae) the correct name for an intriguing and commonly cultivated species. Brittonia 63(4): 500-504.

Ulloa Ulloa, C., Zarucchi, J.L. \& León, B. 2004. Diez años de adiciones a la flora del Perú: 1993-2003. Arnaldoa, edición especial, nov. 2004: 1-242

Thiers, B. 2013. Index Herbariorum: A global directory of public herbaria and associated staff. New York Botanical Garden's Virtual Herbarium. Available at http://www.sweetgum.nybg.org/ih/ (accessed February 5, 2013).

Tokuoka, T. (2007) Molecular phylogenetic analysis of Euphorbiaceae sensu stricto based on plastid and nuclear DNA sequences and ovule and seed character evolution. Journal of Plant Research 120: 511-522.

Valenzuela, L., Calatayud, G., Farfán, J., Humamtupa, I., Monteagudo, A. \& Suclli, E. 2007. Flórula de la Reserva Ecológica Inkaterra, Perú. Missouri Botanical Garden.

Vargas, I.G. 1993. Euphorbiaceae. In: Killeen, T.J., García, E. \& Beck, S.G. (eds.), Guía de Árboles de Bolivia 288-315. Herbario Nacional de Bolivia \& Missouri Botanical Garden, La Paz.

Vásquez, R. 1997. Flórula de las Reservas Biológicas de Iquitos, Perú. Missouri Botanical Garden.

Vásquez, R. 2010. Acalypha. In: Vásquez, R., Rojas, R. \& Werff, H. van der, Flora del Río Cenepa, Amazonas, Perú 1: 633-635. Missouri Botanical Garden.

Wurdack, K.J. \& Davis, C.C. 2009. Malpighiales phylogenetics: Gaining ground on one of the most recalcitrant clades in the angiosperm tree of life. American Journal of Botany 96: 1551-1570.

\section{APPENDIX 1}

Alphabetical list of published names of Acalypha of Peru and Bolivia (accepted in bold)

A. alchorneoides Rusby [=A. diversifolia Jacq.], $\boldsymbol{A}$. alopecuroidea Jacq., A. amblyodonta (Müll. Arg.) Müll. Arg., A. amentacea subsp. wilkesiana (Müll. Arg.) Fosberg [=A. wilkesiana Müll. Arg.], A. argomuelleri Briq., $A$. aronioides Pax \& K. Hoffm., A. arvensis Poepp., $A$. baenitzii Pax [=A. stenoloba Müll. Arg.], A. beckii Cardiel, A. benensis Britton [=A. stricta Poepp.], " $\boldsymbol{A}$. betuloides Pav." nomen nudum $[=\boldsymbol{A}$. diversifolia Jacq.], $\boldsymbol{A}$. boliviensis Müll. Arg., A. bopiana Rusby [=A. stricta Poepp.], A. brachyclada Müll. Arg.[doubtful name], A. brittonii Rusby [=A. stenoloba Müll. Arg.], A. buchtienii Pax $[=A$. hibiscifolia Britton ex Rusby], A. buddleifolia Pax \& K. Hoffm. [=A. argomuelleri Briq.], A. bullata Müll. Arg. [=A. peruviana Müll. Arg.], A. callosa Benth. [=A. mascrostachya Jacq.], A. capillaris Rusby [=A. stenoloba Müll. Arg.], "A. carpinifolia Poepp. ex Seem." nomen nudum [=A. diversifolia Jacq.], A. communis subsp. communis, $A$. communis subsp. saltensis ( $\mathrm{Pax} \& \mathrm{~K}$. Hoffm.) Cardiel \& P. Muñoz, A. communis var. saltensis Pax \& K. Hoffm. [=A. communis subsp. saltensis (Pax \& $\mathrm{K}$. Hoffm.) Cardiel \& P. Muñoz], A. contermina Müll. Arg. [doubtful 
name], A. controversa (Kuntze) K.Schum. [=A. peruviana Müll. Arg.] A. cuneata Poepp., A. cuprea Herzog [doubtful name], A. cuspidata Jacq., A. cuspidata Jacq. var. amblyodonta Müll. Arg. [=A. amblyodonta (Müll. Arg.) Müll. Arg.], A. delicata Cardiel, A. dictyoneura Müll. Arg., A. dictyoneura Müll. Arg. f. reducta Müll. Arg. [=A. dictyoneura Müll. Arg.], A. dictyoneura Müll. Arg. var. reducta (Müll. Arg.) Müll. Arg. [A. dictyoneura Müll. Arg.], A. divaricata Müll. Arg. [=A. aronioides Pax \& K. Hoffm.], A. diversifolia Jacq., A. diversifolia Jacq. var. carpinifolia (Poepp. ex Müll. Arg.) Müll. Arg. [=A. diversifolia Jacq.], A. douilleana Rusby [ $=\boldsymbol{A}$. stenoloba Müll. Arg.], $\boldsymbol{A}$. erosa Rusby [A. cuneata Poepp.], A. eugenifolia Rusby [=A. stenoloba Müll. Arg.], A. flabellifera Rusby [=A. plicata Müll. Arg.], A. foliosa Rusby [=A. macrosytachya Jacq.], $A$. forbesii Moore [=A. infesta Poepp.], A. fulva I.M. Johnst. [=A. plicata Müll. Arg.], A. grandispicata Britton ex Rusby [=A. stenoloba Müll. Arg.], A. herzogiana Pax \& K. Hoffm., A. heteromorpha Rusby [=A. macrostachya Jacq.], $A$. hibiscifolia Britton ex Rusby, $A$. hispida Burm. [=A. hispida Burm.], A. inaequalis Rusby [=A. diversifolia Jacq.], $A$. infesta Poepp. [=A. infesta Poepp.], A. infesta Poepp. var. rotundifolia Müll. Arg. [=A. infesta Poepp.], A. infesta Poepp. var. stenoloba Müll. Arg. [=A. infesta Poepp.], A. jubifera Rusby [doubtful name], A. lechleri Britton ex Rusby [=A. stenoloba Müll. Arg.], A. leptostachya Kunth var. carpinifolia Poepp. ex Müll. Arg. [ $=$ A. diversifolia Jacq.], A. leptostachya Kunth f. diversifolia (Jacq.) Müll. Arg. [=A. diversifolia Jacq.], A. lucida Rusby [=A. stenoloba Müll. Arg.], A. lycioides Pax \& K. Hoffm., A. macbridey I.M. Johnst. [=A. salicifolia Müll. Arg.], A. macrodonta Müll. Arg. [=A. padifolia Kunth], A. macrophylla Ule [=A. stachyura Pax], A. macrostachya Jacq., A. macrostachya Jacq. var. tristis (Poepp.) Müll. Arg. [=A. macrostachya Jacq.], A. machiensis Cardiel \& P. Muñoz, A. mandoni Müll. Arg. [=A. reflexa Müll. Arg.], A. mapirensis $\operatorname{Pax}[=\boldsymbol{A}$. stricta Poepp.], $A$. mapirensis Pax var. pubescens Pax \& K. Hoffm. [=A. stricta Poepp.], A. microyna Poepp. [=A. diversifolia Jacq.], A. multicaulis Müll. Arg., A. neeana Cardiel, $A$. obovata Benth. var. cuneata (Poepp.) J.F. Macbr. [=A. cuneata Poepp.], A. ovata Pax \& K. Hoffm. [=A. stenoloba Müll. Arg.], A. padifolia Kunth, A. paupercula Pax \& K. Hoffm. [=A. poiretii Jacq.], A. peruviana Müll. Arg., A. platyphylla Müll. Arg., A. plicata Müll. Arg., A. poiretii Jacq., $\boldsymbol{A}$. psamofila Cardiel, $\boldsymbol{A}$. reflexa Müll. Arg., "A. rotundifolia Vahl ex Baill." nomen nudum [ $=\boldsymbol{A}$. infesta Poepp.], A. ruiziana Müll. Arg. [=A. padifolia Kunth], A. salicifolia Müll. Arg., A. salicina Cardiel, A. samydifolia Poepp. $[=$ A. diversifolia Jacq.], $\boldsymbol{A}$. scandens Benth., $\boldsymbol{A}$. schultesii Cardiel, A. simplicistila Cardiel, A. soratensis Pax \& K. Hoffm. [=A. mandonii Müll. Arg.], A. stachyura Pax, A. stenoloba Müll. Arg., A. stricta Poepp., A. subandina Ule [=A. platyphylla Müll. Arg.], A. subbullata Pax \& K. Hoffm. [=A. peruviana Müll. Arg.], A. subcastrata F. Arech, A. tarapotensis Müll. Arg. [=A. macrostachya Jacq.], A. tometulosa Ule [=A. stricta Poepp.], A. tristis Poepp. [=A. macrostachya Jacq.], $A$. variabilis Klotzsch ex Baill., $A$. variegata Rusby [=A. stricta Poepp.], A. vermifera Rusby [=A. diversifolia Jacq.], $A$. villosa Jacq., A. villosa Jacq. var. latiuscula Pax \& K. Hoffm. [=A. villosa Jacq.], $A$. villosa Jacq. var. tomentosa Müll. Arg. [=A. villosa Jacq.], $\boldsymbol{A}$. wilkesiana Müll. Arg., A. williamsii Rusby (1912) [=A. macrostachya Jacq.], A. williamsii Rusby (1920) [=A. villosa Jacq.], Ricinocarpus alopecuroides (Jacq.) Kuntze [=A. alopecuroidea Jacq.], R. amblyodontus (Müll. Arg.) Kuntze [=A. amblyodonta (Müll. Arg.) Müll. Arg.], $R$. arvensis (Poepp.) Kuntze $[=\boldsymbol{A}$. arvensis Poepp.], $R$. boliviensis (Müll. Arg.) Kuntze $[=\boldsymbol{A}$. boliviensis Müll. Arg.], $R$. controversus Kuntze [=A. peruviana Müll. Arg.], $R$. cuneatus (Poepp.) Kuntze [=A. cuneata Poepp.], R. cuspidatus (Jacq.) Kuntze $[=\boldsymbol{A}$. cuspidata Jacq.], $R$. cuspidatus (Jacq,) Kuntze var. glandulosus Kuntze [=A. plicata Müll. Arg.], R. dictyoneurus (Müll. Arg.) Kuntze [=A. dictyoneura Müll. Arg.], R. divaricatus (Müll. Arg.) Kuntze [=A. aronioides Pax \& K. Hoffm.], R. diversifolius (Jacq.) Kunth [=A. diversifolia Jacq.], $R$. gracilis var arboreus Kuntze [=A. stenoloba Müll. Arg.], R. hispidus (Burm.) Kuntze [=A. hispida Burm.], R. infestus (Poepp.) Kuntze [=A. infesta Poepp.], R. macrodontus (Müll. Arg.) Kuntze [=A. padifolia Kunth], R. macrostachyus (Jacq.) Kuntze $[=\boldsymbol{A}$. macrostachya Jacq.], $R$. mandoni (Müll. Arg.) Kuntze [=A. mandoni Müll. Arg.], R. multicaulis (Müll. Arg.) Kuntze [=A. multicaulis Müll. Arg.], R. padifolius (Kunth) Kuntze [=A. padifolia Kunth], R. peruvianus (Müll. Arg.) Kuntze [=A. peruviana Müll. Arg.], R. plicatus (Müll. Arg.) Kuntze [=A. plicata Müll. Arg.], R. poiretii (Jacq.) Kuntze [=A. poiretii Jacq.], R. salicifolius (Müll. Arg.) Kuntze [=A. salicifolia Müll. Arg.], $R$. samydifolius (Poepp.) Kuntze [=A. diversifolia Jacq.], $R$. scandens (Benth.) Kuntze [=A. scandens Benth.], R. tarapotensis (Müll. Arg.) Kuntze [=A. macrostachya Jacq.], $R$. villosus (Jacq.) Kuntze $[=\boldsymbol{A}$. villosa Jacq.], R. wilkesianus (Müll. Arg.) Kuntze [=A. wilkesiana Müll. Arg.].

Associate Editor: Ricarda Riina Received: 24-IX-2013 Accepted: 13-XII-2013 